\title{
The spectral shift function for planar obstacle scattering at low energy
}

\author{
I McGillivray \\ School of Mathematics \\ University of Bristol \\ University Walk \\ Bristol BS8 1TW \\ United Kingdom \\ e maiemg@bristol.ac.uk \\ $\mathrm{t}+44(0) 1173311663$ \\ $\mathrm{f}+44(0) 1179287999$
}

\begin{abstract}
Let $H$ signify the free non-negative Laplacian on $\mathbb{R}^{2}$ and $H_{Y}$ the non-negative Dirichlet Laplacian on the complement $Y$ of a nonpolar compact subset $K$ in the plane. We derive the low-energy expansion for the Krein spectral shift function (scattering phase) for the obstacle scattering system $\left\{H_{Y}, H\right\}$ including detailed expressions for the first three coefficients. We use this to investigate the large time behaviour of the expected volume of the pinned Wiener sausage associated to $K$.
\end{abstract}

Key words: obstacle scattering, regularised heat-trace, Krein spectral shift function, scattering phase, pinned Wiener sausage, Brownian bridge

Mathematics Subject Classification 2010: 60J65, 35B40, 47A40

\section{Introduction}

Given a nonpolar compact subset $K$ in the plane, we consider the exterior domain $Y$ complementary to $K$. Let $\mathscr{H}$ stand for the Hilbert space $L^{2}\left(\mathbb{R}^{2}, m\right)$ where $m$ signifies the Lebesgue measure. The (non-negative) Laplacian acting in $\mathscr{H}$ will be denoted $H$ while $H_{Y}$ denotes the (non-negative) Dirichlet Laplacian acting in $L^{2}(Y, m)$. The operator $J$ embeds $L^{2}(Y, m)$ into $\mathscr{H}$ through extending by zero on $Y$. Let $\xi(\lambda)$ stand for the Krein spectral shift function (scattering phase) for the pair $\left\{H_{Y}, H\right\}$. The following trace formula then holds

$$
\operatorname{Tr}\left[J g\left(H_{Y}\right) J^{*}-g(H)\right]=\int_{0}^{\infty} g^{\prime}(\lambda) \xi(\lambda) d \lambda
$$

for any function $g: \mathbb{R} \rightarrow \mathbb{R}$ of the form

$$
g(\lambda)= \begin{cases}f\left(e^{-\lambda}\right) & \text { for } \quad \lambda>0 \\ 0 & \text { for } \quad \lambda \leq 0\end{cases}
$$

where $f: \mathbb{R} \rightarrow \mathbb{R}$ is a continuously differentiable function with $f^{\prime} \in W^{1,2}(\mathbb{R})$. In this paper, we study the asymptotic behaviour of $\xi(\lambda)$ for small $\lambda$. Our main result is this.

Theorem 1.1. Let $K$ be a nonpolar compact subset of $\mathbb{R}^{2}$. Let $l \in \mathbb{N}$. There exist $\xi_{0}^{k} \in \mathbb{R}$ $(-\infty<k \leq-1)$ such that

$$
\xi(\lambda)=\sum_{k=-l}^{-1} \xi_{0}^{k}(-\log \lambda)^{k}+o\left((-\log \lambda)^{-l}\right)
$$

as $\lambda \downarrow 0$. The first three coefficients are given by

(i) $\xi_{0}^{-1}=1$; 
(ii) $\xi_{0}^{-2}=C(K)-\log 4+2 \gamma$;

(iii) $\xi_{0}^{-3}=(C(K)-\log 4+2 \gamma)^{2}-\frac{\pi^{2}}{3}$.

The quantity $C(K)$ is related to the Robin constant $R(K)$ of $K$ via the relation $C(K)=-4 \pi R(K)$; $\gamma$ stands for Euler's constant. We remark that the leading order expansion for $\xi(\lambda)$ has been derived in [15]. The counterpart to this result in higher dimensions may be found in [18], [19].

The study is motivated in part by its relevance to a problem in probability theory. The pinned Wiener sausage $S(t, \omega)$ refers to the random set swept out by a compact set $K$ in the plane as it is transported along a Brownian loop $\omega:[0, t] \rightarrow \mathbb{R}^{2}$ by rigid motion. In detail,

$$
S(t, \omega):=\bigcup_{0 \leq s \leq t}(\omega(s)+K) \subseteq \mathbb{R}^{2}
$$

its area is denoted $|S(t, \omega)|$. Introduce the expected area via

$$
\gamma(t):=\mathbb{E}_{0,0}^{0, t}|S(t, \omega)|
$$

where $\mathbb{P}_{0,0}^{0, t}$ signifies the Brownian bridge measure on loop space associated to the Laplacian $\Delta$. We derive an asymptotic expansion for the above quantity in the large time régime.

Theorem 1.2. Let $K$ be a nonpolar compact subset of $\mathbb{R}^{2}$. Let $l \in \mathbb{N}$. Then there exist $\gamma_{0}^{k} \in \mathbb{R}$ $(\mathbb{Z} \ni k \leq-1)$ such that

$$
\gamma(t)=\sum_{k=-l}^{-1} \gamma_{0}^{k} t(\log t)^{k}+o\left(t(\log t)^{-l}\right)
$$

as $t \rightarrow \infty$. The first three coefficients are given by

(i) $\gamma_{0}^{-1}=4 \pi$;

(ii) $\gamma_{0}^{-2}=4 \pi\{C(K)+\gamma-\log 4\}$;

(iii) $\gamma_{0}^{-3}=4 \pi\left\{(C(K)+\gamma-\log 4)^{2}-\frac{\pi^{2}}{6}\right\}$.

This result was conjectured in [5]. This latter work derives the first order asymptotic expansion of $\gamma(t)$ for an arbitrary nonpolar compact $K$. It also obtains the third order asymptotic series as above for the particular case in which $K=K_{a}$ is a closed disc with radius $a>0$.

It is interesting to compare the behaviour of $\gamma(t)$ with the related functional $\beta(t):=\mathbb{E}_{0}|S(t, \omega)|$. Here $\omega:[0, \infty) \rightarrow \mathbb{R}^{2}$ is a Brownian path in $\mathbb{R}^{2}$ and $\mathbb{P}_{0}$ stands for the Wiener measure on path space associated to $\Delta$. In the large time régime

$$
\beta(t)=\sum_{k=-l}^{-1} \beta_{0}^{k} t(\log t)^{k}+o\left(t(\log t)^{-l}\right)
$$

with explicit expressions for the first three coefficients according to [13. This last expansion extends the work of [23] which detailed the second order expansion. The series for $\gamma(t)$ and $\beta(t)$ agree to leading order. For the lower order terms we have that

$$
\beta_{0}^{-2}=4 \pi\{C(K)+1+\gamma-\log 4\} \quad \text { while } \quad \beta_{0}^{-3}=4 \pi\left\{(C(K)+1+\gamma-\log 4)^{2}-\frac{\pi^{2}}{6}\right\} .
$$

The analogous problem for $\gamma$ in higher dimensions has been treated in [18, [19]. This problem originated in the calculation of the specific heat of a quantum system of obstacles $K$ at low temperature [28]. 
In Section 2 we introduce the trace formula (1.1) and relate the Krein spectral shift function $\xi(\lambda)$ to the scattering matrix $S(\lambda)$ for the system $\left\{H_{Y}, H\right\}$ via the Birman-Krein formula. We show that this relation holds in particular on an interval of the form $(0, \delta)$, including the case when $K$ does not have a connected complement. In Section 3 we derive a number of prerequisite results in logarithmic potential theory.

In order to construct the scattering matrix $S(\lambda)$ it is necessary to invert the operator

$$
I+R^{(-1)}(\mu-\imath 0) V
$$

in $B\left(\mathscr{H}_{-s}\right)$ for $\lambda>0$ in a neighbourhood of $\lambda=0$; here, $\mu$ relates to $\lambda$ via $\mu=(\lambda+1)^{-1}$. To explain terminology briefly,

$$
V=J R_{Y}(-1) J^{*}-R(-1)
$$

denotes the difference between the Dirichlet and free resolvents; while $R^{(-1)}(\cdot)$ signifies the resolvent of $R(-1)$. Also, $\mathscr{H}_{-s}$ refers to a weighted Hilbert space. The operator in (1.2) explodes on the complement of a hyperspace in $\mathscr{H}_{-s}$. This complication is absent in higher dimensions; it presents the salient technical difficulty of the paper. This is tackled in Sections 4 and 6 .

Section 6 continues with a small energy expansion of the scattering matrix $S(\lambda)$ in a double-series akin to expansions obtained in [4, [14. A lattice-point counting lemma in Section 5 plays a role in establishing summability of the double-series. Section 6 culminates in the proof of the expansion given in Theorem 1.1 The detailed derivation of the coefficients is left to Section 7 . The application Theorem 1.2 is proved in Section 8. The Appendix includes the proofs of several results from [18.

\section{The trace formula}

The free Laplacian. Let $\mathscr{H}$ stand for the complex Hilbert space $L^{2}\left(\mathbb{R}^{2}, m\right)$ based on Lebesgue measure $m$ with inner product $(\cdot, \cdot)$ linear in the first factor. We refer to the non-negative Laplacian $-\Delta$ in $\mathscr{H}$ by $H$. Its resolvent $R(\zeta):=(H-\zeta)^{-1}(\zeta \in \mathbb{C} \backslash[0, \infty))$ has convolution kernel $k(x ; \zeta)$ given by

$$
k(x ; \zeta):=\frac{\imath}{4} H_{0}^{(1)}\left(\zeta^{1 / 2}|x|\right)
$$

where $H_{0}^{(1)}$ is the first Hankel function of order 0 . The condition $\Im \zeta^{1 / 2}>0$ specifies the branch of $\zeta^{1 / 2}$. For the sake of completeness, we recall that

$$
\begin{aligned}
& H_{0}^{(1)}(z)=1+\frac{2}{\pi} \gamma \imath-\left\{1+\frac{2}{\pi} \imath(\gamma-1)\right\} \frac{z^{2} / 4}{(1 !)^{2}}+\left\{1+\frac{2}{\pi} \imath\left(\gamma-1-\frac{1}{2}\right)\right\} \frac{\left(z^{2} / 4\right)^{2}}{(2 !)^{2}}+\cdots \\
& +\frac{2}{\pi} \gamma \imath \log (z / 2)\left\{1-\frac{z^{2} / 4}{(1 !)^{2}}+\frac{\left(z^{2} / 4\right)^{2}}{(2 !)^{2}}-\cdots\right\} \quad(z \in \mathbb{C} \backslash[0, \infty))
\end{aligned}
$$

as in [1] 9.1.3, 9.1.12, 9.1.13. The logarithm Log refers to the principal branch of the logarithm.

Let us introduce constants

$$
\begin{aligned}
& a_{j}:= \begin{cases}(1 / 2 \pi)(\log 2-\gamma)+\imath / 4, \\
\left\{\frac{1}{2 \pi}\left(\log 2-\gamma-\sum_{k=1}^{j} \frac{1}{k}\right)+\frac{\imath}{4}\right\} \frac{(-1)^{j}}{4^{j}(j !)^{2}}, & j \geq 1 ;\end{cases} \\
& b_{j}:= \begin{cases}-1 / 2 \pi, & j=0, \\
\frac{(-1)^{j+1}}{2 \pi} \frac{1}{4^{j}(j !)^{2}}, & j \geq 1 ;\end{cases} \\
& c_{j}:=\frac{1}{4 \pi} \frac{(-1)^{j}}{4^{j}(j !)^{2}}, \quad j \geq 0 .
\end{aligned}
$$


Put

$$
k_{j}^{0}(x)=\left\{a_{j}+b_{j} \log |x|\right\}|x|^{2 j} \text { and } k_{j}^{1}(x)=c_{j}|x|^{2 j} \quad\left(x \in \mathbb{R}^{2} \backslash\{0\}\right) .
$$

Then

$$
k(x ; \zeta)=\sum_{j=0}^{\infty} \sum_{\varepsilon=0}^{1} \zeta^{j} \eta^{\varepsilon} k_{j}^{\varepsilon}(x) \quad\left(x \in \mathbb{R}^{2} \backslash\{0\}\right)
$$

with $\eta:=-2 \log \zeta^{1 / 2}$.

Define $\langle x\rangle:=\left(1+|x|^{2}\right)^{1 / 2}$ for $x \in \mathbb{R}^{2}$. The weighted $L^{2}$-space $\mathscr{H}_{s}(s \in \mathbb{R})$ is defined by $\mathscr{H}_{s}:=$ $\left\{u:\langle\cdot\rangle^{s} u \in \mathscr{H}\right\}$. Considered as Banach spaces, the dual space of $\mathscr{H}_{s}$ is $\mathscr{H}_{-s}$. We write $\langle\cdot, \cdot\rangle$ for the corresponding duality pairing.

According to [3] Theorem 4.1,

$$
R(\lambda \pm \imath 0):=\lim _{\varepsilon \downarrow 0} R(\lambda \pm \imath \varepsilon)
$$

exists in $B\left(\mathscr{H}_{s}, \mathscr{H}_{-s}\right)$ for any $s>1 / 2$ and $\lambda>0$, with convergence in the uniform operator topology. Further,

Theorem 2.1. Let $l \in \mathbb{N}_{0}$ and $s>2 l+1$. Then for $\zeta \in \mathbb{C} \backslash[0, \infty)$,

$$
\left\|R(\zeta)-\sum_{j=0}^{l} \sum_{\varepsilon=0}^{1} \zeta^{j} \eta^{\varepsilon} K_{j}^{\varepsilon}\right\|_{B\left(\mathscr{H}_{s}, \mathscr{H}_{-s}\right)}=o\left(|\zeta|^{l}\right)
$$

as $\zeta \rightarrow 0$ where $K_{j}^{\varepsilon}$ is the operator with convolution kernel $k_{j}^{\varepsilon}$.

This is proved in the Appendix (see also Proposition 3.7 in [18]).

The modified resolvent $R^{(-1)}(\zeta)$ is the resolvent of $R(-1)$. It relates to the resolvent of $H$ via

$$
R^{(-1)}\left((1+\zeta)^{-1}\right)=-(1+\zeta)(I+(1+\zeta) R(\zeta)), \quad \zeta \in \mathbb{C} \backslash[0, \infty) .
$$

The auxiliary Hilbert space $L^{2}\left(S^{1}, \sigma\right)$ is denoted by $\mathfrak{h}$. Let $U: \mathscr{H} \rightarrow L^{2}((0, \infty) ; \mathfrak{h})$ be the spectral representation of $H$. Then for any $u \in \mathscr{H}_{s}$ and $\lambda>0$,

$$
U(\lambda) u(\omega)=(1 / \sqrt{2}) \mathscr{F} u\left(\lambda^{1 / 2} \omega\right) \quad\left(\omega \in S^{1}\right)
$$

provided that $s>1$. Here, $\mathscr{F}$ stands for the Fourier transform

$$
\mathscr{F} u(\xi)=\widehat{u}(\xi):=(2 \pi)^{-1} \int_{\mathbb{R}^{2}} e^{-\imath \xi \cdot x} u(x) m(d x) .
$$

The following lemma is proved in the Appendix (see also [18] Lemma 3.9).

Lemma 2.1. Fix $l \in \mathbb{N}_{0}$ and $s>l+1$. Then

$$
\left\|U(\lambda)-\sum_{j=0}^{l}\left(\imath \lambda^{1 / 2}\right)^{j} U_{j}\right\|_{\mathfrak{S}_{2}\left(\mathscr{H}_{s}, \mathfrak{h}\right)}=o\left(\lambda^{l / 2}\right)
$$

as $\lambda \downarrow 0$. The operator $U_{j}$ has kernel

$$
u_{j}(\omega, x)=\frac{1}{\sqrt{2}}(2 \pi)^{-1} \frac{(-1)^{j}}{j !}(\omega \cdot x)^{j} .
$$


Incidentally, the notation $\mathfrak{S}_{2}\left(\mathscr{H}_{s}, \mathfrak{h}\right)$ refers to the collection of operators from $\mathscr{H}_{s}$ to $\mathfrak{h}$ of HilbertSchmidt type.

Let $U^{(-1)}: \mathscr{H} \rightarrow L^{2}((0,1) ; \mathfrak{h})$ be the spectral representation of $R(-1)$. Then $U$ and $U^{(-1)}$ are related via

$$
U^{(-1)}(\mu)=(\lambda+1) U(\lambda)
$$

We use the notation

$$
\mu=(\lambda+1)^{-1}
$$

and this is used routinely in the sequel. Let $l \in \mathbb{N}_{0}$ and $s>l+1$. From Lemma 2.1 we derive

$$
U^{(-1)}(\mu)=\sum_{j=0}^{2 l}\left(\imath \lambda^{1 / 2}\right)^{j} U_{j}^{(-1)}+o\left(\lambda^{l}\right)
$$

in $\mathfrak{S}_{2}\left(\mathscr{H}_{s}, \mathfrak{h}\right)$ as $\lambda \downarrow 0$ where

$$
\begin{aligned}
& U_{0}^{(-1)}=U_{0}, \\
& U_{1}^{(-1)}=U_{1}, \\
& U_{j}^{(-1)}=U_{j}-U_{j-2} \text { for } j \geq 2 .
\end{aligned}
$$

The spectral shift function. Let $K$ be a nonpolar compact subset of $\mathbb{R}^{2}$. Its complement will be denoted by $Y$. Let $H_{Y}$ refer to the non-negative Dirichlet Laplacian on $L^{2}(Y, m)$. The semigroup difference

$$
J e^{-H_{Y}} J^{*}-e^{-H} \in \mathfrak{S}_{1}(\mathscr{H})
$$

is trace class [24]. Let $\xi\left(\lambda, e^{-H_{Y}}, e^{-H}\right)$ be the spectral shift function for the pair $\left\{e^{-H_{Y}}, e^{-H}\right\}$ (31] Theorem 8.2.1). Define

$$
\xi(\lambda)=\xi\left(\lambda, H_{Y}, H\right):= \begin{cases}-\xi\left(e^{-\lambda}, e^{-H_{Y}}, e^{-H}\right), & \lambda \geq 0, \\ 0, & \lambda<0 .\end{cases}
$$

By 31] Theorem 8.2.1,

$$
\xi \in L^{1}\left(\mathbb{R} ; e^{-|\lambda|} d \lambda\right)
$$

By [31] Theorem 8.3.3 and the paragraph following it, we may write

$$
\operatorname{Tr}\left[J g\left(H_{Y}\right) J^{*}-g(H)\right]=\int_{0}^{\infty} g^{\prime}(\lambda) \xi(\lambda) d \lambda
$$

for any function $g: \mathbb{R} \rightarrow \mathbb{R}$ of the form

$$
g(\lambda)= \begin{cases}f\left(e^{-\lambda}\right) & \text { for } \quad \lambda>0 \\ 0 & \text { for } \quad \lambda \leq 0\end{cases}
$$

where $f: \mathbb{R} \rightarrow \mathbb{R}$ is a continuously differentiable function with $f^{\prime} \in W^{1,2}(\mathbb{R})$. In particular, given $t>3 / 2$ we can find a continuously differentiable function $f$ such that $f(\lambda)=\lambda^{t}$ for $0 \leq \lambda \leq 1$ and $f^{\prime} \in W^{1,2}(\mathbb{R})$. We then have

$$
\operatorname{Tr}\left[J e^{-t H_{Y}} J^{*}-e^{-t H}\right]=-\int_{0}^{\infty} t e^{-t \lambda} \xi(\lambda) d \lambda
$$

for $t>3 / 2$. 
Let $Y_{e}$ stand for the unbounded connected component of $Y$ and set $Y_{b}:=Y \backslash Y_{e}$. In case $Y_{b} \neq \emptyset$, we differentiate between $H_{e}$ resp. $H_{b}$, the non-negative Dirichlet Laplacians on $Y_{e} \operatorname{resp} . Y_{b}$. The spectrum $\sigma\left(H_{b}\right)$ of $H_{b}$ is discrete. Let $\xi_{e}(\lambda)=\xi\left(\lambda, H_{Y_{e}}, H\right)$ be the spectral shift function for the pair $\left\{H_{Y_{e}}, H\right\}$. Denote by

$$
N_{b}(\lambda):=\sum_{\sigma\left(H_{b}\right) \ni \nu<\lambda} m(\nu)
$$

the spectral counting function for $H_{b}$; here, $m(\nu)$ stands for the geometric multiplicity of $\nu \in$ $\sigma\left(H_{b}\right)$.

Lemma 2.2. It holds that

(i) $\xi(\lambda)=\xi_{e}(\lambda)+N_{b}(\lambda)$ for a.e. $\lambda>0$;

(ii) $\xi$ admits an a.e.-version that is real analytic on $(0, \infty) \backslash \sigma\left(H_{b}\right)$.

Finally, with $\xi$ denoting this version,

(iii) $\xi(\lambda) \rightarrow 0$ as $\lambda \downarrow 0$.

Proof. First note that both $\xi$ and $\xi_{e}$ satisfy (2.13). For $t>3 / 2$,

$$
\int_{0}^{\infty} e^{-t \lambda} \xi(\lambda) d \lambda=t^{-1} \operatorname{Tr}\left[e^{-t H}-J_{e} e^{-t H_{Y_{e}}} J_{e}^{*}\right]-t^{-1} \operatorname{Tr}\left[e^{-t H_{Y_{b}}}\right]=\int_{0}^{\infty} e^{-t \lambda}\left\{\xi_{e}(\lambda)+N_{b}(\lambda)\right\} d \lambda
$$

where the Weyl asymptotics of $N_{b}(\cdot)$ ensure the absolute integrability of the second integrand. Item (i) follows by the inversion formula for the Laplace-Lebesgue integral ([30] Theorem VII.6a). Parts (ii) and (iii) follow from [16] Lemmas 3.2 and 3.4 and (i).

The scattering matrix. In virtue of (2.11) the scattering operator $S\left(e^{-H_{Y}}, e^{-H}, J\right)$ for the pair $\left\{e^{-H_{Y}}, e^{-H}\right\}$ exists and is unitary on $\mathscr{H}$ by 31] Theorem 6.2.1 and Corollary 2.4.2. By the invariance principle ([31] Theorem 6.2.5), the scattering operator $S\left(R_{Y}(-1), R(-1), J\right)$ exists and $S\left(R_{Y}(-1), R(-1), J\right)=S\left(e^{-H_{Y}}, e^{-H}, J\right)$. As the scattering operators commute with the corresponding spectral projectors ([31] Theorem 2.1.4 and 1.5.1) we have the representation

$$
S\left(\lambda, e^{-H_{Y}}, e^{-H}, J\right)=S\left(\varphi(\lambda), R_{Y}(-1), R(-1), J\right) \quad \text { a.e. } \lambda>0
$$

where $\varphi:(0,1) \rightarrow(0,1) ; \lambda \mapsto(-\log \lambda+1)^{-1}$. By the Birman-Kreǐn formula (31 Theorem 8.4.1),

$$
\begin{aligned}
e^{2 \pi \imath \xi(\lambda)} & =\operatorname{Det}\left(S\left(e^{-\lambda}, e^{-H_{Y}}, e^{-H}, J\right)\right) \\
& =\operatorname{Det}\left(S\left(\mu, R_{Y}(-1), R(-1), J\right)\right) \quad \text { a.e. } \lambda>0 .
\end{aligned}
$$

We now derive a representation formula for $S\left(\mu, R_{Y}(-1), R(-1), J\right)$.

Set

$$
V:=J R_{Y}(-1) J^{*}-R(-1)
$$

Then

Theorem 2.2. For each $s>0, V$ admits a bounded extension from $\mathscr{H}_{-s}$ to $\mathscr{H}_{s}$ and $V=V^{*} \in$ $\mathfrak{S}_{\infty}\left(\mathscr{H}_{-s}, \mathscr{H}_{s}\right)$ is compact.

This result is proved in the Appendix (see also [18] Theorem 2.1). Given $s>1 / 2$ and $\mu \in(0,1)$, define

$$
\mathscr{H}_{s}^{\mu}:=\left\{f=(R(-1)-\mu) u: u \in \mathscr{H}_{s}\right\}
$$


Lemma 2.3. Let $s>1 / 2$ and $\mu \in(0,1)$. We have

(i) $R(-1) \in B\left(\mathscr{H}_{s}, \mathscr{H}_{s}\right)$;

(ii) $\mathscr{H}_{s}^{\mu}$ is a proper subspace of $\mathscr{H}_{s}$;

(iii) the identity

$$
R^{(-1)}(\mu \pm \imath 0)(R(-1)-\mu)=I
$$

holds on $\mathscr{H}_{s}$.

Proof. (i) From the identity $\langle x\rangle^{s} \leq 2^{s}\left\{\langle y\rangle^{s}+\langle x-y\rangle^{s}\right\}$ obtain

$$
\langle x\rangle^{s} k(x-y ;-1)\langle y\rangle^{-s} \leq k(x-y ;-1)+\langle x-y\rangle^{s} k(x-y ;-1) \quad(x \neq y) .
$$

The latter kernel is integrable by [25] 3.6, so defines a bounded convolution operator on $\mathscr{H}$ by Young's inequality 8 .

(ii) Let us introduce the Sobolev space $W_{s}:=\left\{u: \widehat{u} \in \mathscr{H}_{s}\right\}$. Let $\tau: W_{s} \rightarrow L^{2}\left(S_{\lambda}^{1}, \sigma\right)$ stand for the restriction mapping ([22] Theorem IX.39). Then $\tau \widehat{f}=0$ for any $f \in \mathscr{H}_{s}^{\mu}$. The function $f=e^{-|\cdot|^{2} / 2} \in \mathscr{H}_{s}$ does not satisfy this property as $\widehat{f}=f([17]$ Theorem 5.2).

(iii) Let $u \in \mathscr{H}_{s}$ and $f:=(R(-1)-\mu) u$. Then

$$
\begin{aligned}
\left\|u-R^{(-1)}(\mu-\imath 0) f\right\|_{\mathscr{H}_{-s}} & =\lim _{\varepsilon \downarrow 0}\left\|u-R^{(-1)}(\mu-\imath \varepsilon) f\right\|_{\mathscr{H}_{-s}} \\
& =\lim _{\varepsilon \downarrow 0}\left\|u-R^{(-1)}(\mu-\imath \varepsilon)[R(-1)-(\mu-\imath \varepsilon)-\imath \varepsilon] u\right\|_{\mathscr{H}_{-s}} \\
& =\lim _{\varepsilon \downarrow 0} \varepsilon\left\|R^{(-1)}(\mu-\imath \varepsilon) u\right\|_{\mathscr{H}_{-s}} \\
& =0
\end{aligned}
$$

and similar with the opposite sign.

Theorem 2.3. Let $s>1 / 2$.

(i) Assume that $Y_{b}=\emptyset$. For any $\mu \in(0,1)$, the compact operator $V R^{(-1)}(\mu \pm \imath 0)$ acting in $B\left(\mathscr{H}_{s}\right)$ does not have eigenvalue -1 .

(ii) Assume that $Y_{b} \neq \emptyset$ and $\lambda \notin \sigma\left(H_{b}\right)$. Then $V R^{(-1)}(\mu \pm \imath 0)$ acting in $B\left(\mathscr{H}_{s}\right)$ does not have eigenvalue -1 .

(iii) Assume that $Y_{b} \neq \emptyset$ and $\lambda \in \sigma\left(H_{b}\right)$. Then $V R^{(-1)}(\mu \pm \imath 0)$ acting in $B\left(\mathscr{H}_{s}\right)$ has eigenvalue -1 .

Proof. (i) Suppose that $V R^{(-1)}(\mu-\imath 0) f=-f$ for some $f \in \mathscr{H}_{s}$. Set $u:=R^{(-1)}(\mu-\imath 0) f$. Argue as in [7] Lemma 4.4 to conclude that $u \in \mathscr{H}$ and that $R_{Y}(-1) u=\mu u$. Put $f:=\Delta u+\lambda u \in \mathscr{D}^{\prime}\left(\mathbb{R}^{2}\right)$ with $\lambda:=-1+1 / \mu$. Then $f=0$ on $Y$ because $u$ is a weak solution of $\Delta u+\lambda u=0$ there. By elliptic regularity [25] Proposition 3.9.1, $u$ is smooth on $Y$. Adapting the argument in [26] Lemma 1.2 to the $d=2$ case, conclude that $u$ vanishes on the complement of some ball $B(0, r)$. The unique continuation property (11] Theorem 5.1, for example) ensures that $u$ vanishes throughout $Y$. The proof of (ii) is similar.

(iii) Let $\varphi \in L^{2}\left(Y_{b}\right)$ be an eigenfunction of $H_{b}$ corresponding to $\lambda$. Let $u \in \mathscr{H}_{s}$ be the extension of $\varphi$ by 0 . Then $f:=[R(-1)-\mu] u \in \mathscr{H}_{s}$ by Lemma $2.3(i)$. Also, $R^{(-1)}(\mu-\imath 0) f=u$ by Lemma 2.3 (iii). In an obvious notation,

$$
V u=\left[R_{Y_{b}}(-1) \oplus R_{Y_{e}}(-1)-R(-1)\right] u=-[R(-1)-\mu] u=-f ;
$$

that is, $V R^{(-1)}(\mu-\imath 0) f=-f$. 
For $\lambda \in(0, \infty) \backslash \sigma\left(H_{b}\right)$,

$$
\exists\left(I+V R^{(-1)}(\mu+\imath 0)\right)^{-1} \in B\left(\mathscr{H}_{s}\right)
$$

by the Fredholm alternative. As in 31 Theorem 5.7.1' (with $\mathfrak{G}=\mathscr{H}_{s}$ for $s>1 / 2$ and $G: \mathscr{H} \rightarrow$ $\left.\mathfrak{G} ; f \mapsto\langle\cdot\rangle^{-2 s} f\right)$ the scattering matrix for $\left\{R_{Y}(-1), R(-1)\right\}$ can be represented

$$
S\left(\mu, R_{Y}(-1), R(-1), J\right)=I-2 \pi \imath U^{(-1)}(\mu)\left(I+V R^{(-1)}(\mu+\imath 0)\right)^{-1} V U^{(-1)}(\mu)^{*}, \quad \text { a.e. } \lambda \in(0, \infty) \backslash \sigma\left(H_{b}\right) .
$$

Let $S(\cdot)$ stand for the (adjoint) scattering matrix for $\left\{H, H_{Y}\right\}$,

$$
S(\lambda)=I+2 \pi \imath U^{(-1)}(\mu) V\left(I+R^{(-1)}(\mu-\imath 0) V\right)^{-1} U^{(-1)}(\mu)^{*} \in B(\mathfrak{h})
$$

with $\lambda \in(0, \infty) \backslash \sigma\left(H_{b}\right)$. The $t$-matrix is characterised by the relation $S(\lambda)=I+T(\lambda)$. From (2.17),

$$
e^{-2 \pi \imath \xi(\lambda)}=\operatorname{Det} S(\lambda) \quad \text { a.e. } \lambda>0 .
$$

\section{Some logarithmic potential theory}

For brevity, we use the notation $g^{\lambda}$ to stand for the resolvent operator $R(-\lambda)$ with $\lambda>0 ; g^{\lambda}(\cdot)$ stands for the coresponding convolution kernel. If $\lambda=0$ the notation $g$ is sometimes used. For $z \in \mathbb{C} \backslash(-\infty, 0]$ define

$$
b(z):=H_{0}^{(1)}(z)-1-\frac{2 \imath}{\pi}\{\log (z / 2)+\gamma\} .
$$

Given $0<\delta<1$ there exists a finite constant $c$ such that

$$
|b(z)| \leq C|z|^{2}(-\log |z|) \text { for }|z| \leq \delta .
$$

The logarithmic potential kernel is defined by

$$
k(x):=(1 / 2 \pi) \log (1 /|x|) \quad\left(x \in \mathbb{R}^{2} \backslash\{0\}\right) .
$$

From (2.1), the kernel $g^{\lambda}(\cdot)$ may be decomposed in terms of (3.3) and (3.1) as

$$
g^{\lambda}(x)=a_{0}-\imath \pi c_{0}-c_{0} \log \lambda+k(x)+r\left(\lambda^{1 / 2}|x|\right) \quad\left(x \in \mathbb{R}^{2} \backslash\{0\}\right)
$$

with $a_{0}$ and $c_{0}$ as in (2.3) and (2.5), and

$$
r(x):=(\imath / 4) b(\imath x) .
$$

Fix a unit vector $u$ in $\mathbb{R}^{2}$. The regularised resolvent kernel $k^{\lambda}(\cdot)$ is given by

$$
k^{\lambda}(x):=g^{\lambda}(x)-g^{\lambda}(u) \quad\left(x \in \mathbb{R}^{2} \backslash\{0\}\right) .
$$

From (3.4),

$$
g^{\lambda}(u)=a_{0}-\imath \pi c_{0}-c_{0} \log \lambda+r\left(\lambda^{1 / 2}\right),
$$

and hence

$$
k^{\lambda}(x)-k(x)=r\left(\lambda^{1 / 2}|x|\right)-r\left(\lambda^{1 / 2}\right) \quad\left(x \in \mathbb{R}^{2} \backslash\{0\}\right) .
$$

The operators with convolution kernels $k^{\lambda}(\cdot)$ resp. $k(\cdot)$ will be denoted by $k^{\lambda}$ resp. $k$. We use the notation $r^{\lambda}$ to refer to the operator with convolution kernel $r\left(\lambda^{1 / 2}|x|\right)$. 
Corollary 3.1. Let $s>1$. Then $k^{\lambda} \rightarrow k$ in $B\left(\mathscr{H}_{s}, \mathscr{H}_{-s}\right)$ as $\lambda \downarrow 0$.

Proof. We may write

$$
k^{\lambda}-k=g^{\lambda}-\eta K_{0}^{1}-K_{0}^{0}-r\left(\lambda^{1 / 2}\right)\langle\cdot, 1\rangle 1
$$

with $\eta$ as before given by $\eta=-2 \log (-\lambda)^{1 / 2}=-\log \lambda-\imath \pi$. Now apply Theorem 2.1 and (3.2).

Theorem 3.1. Let $s>1$. Then

(i) $k: \mathscr{H}_{s} \rightarrow C\left(\mathbb{R}^{2}\right)$;

(ii) $r^{\lambda}: \mathscr{H}_{s} \rightarrow C\left(\mathbb{R}^{2}\right)$ for each $\lambda>0$;

(iii) $g^{\lambda}(u) r^{\lambda} f \rightarrow 0$ locally uniformly on $\mathbb{R}^{2}$ as $\lambda \downarrow 0$ for each $f \in \mathscr{H}_{s}$.

Proof. (i) Define

$$
k_{1} f(x):=-(1 / 2 \pi) \int_{B(x, 1)} \log |x-y| f(y) m(d y),
$$

and likewise for $k_{2}$ but with $B(x, 1)$ replaced by its complement $B(x, 1)^{c}$. Note that

$$
\left|\int_{B(x, r)} f(y) m(d y)\right| \leq \sqrt{\pi}\|f\|_{\mathscr{H}_{s}} r
$$

for $f \in \mathscr{H}_{s}$. As in [2] Lemma 3.1.1 (b),

$$
k_{1} f(x)=(1 / 2 \pi) \int_{0}^{1} \int_{B(x, r)} f(y) m(d y) \frac{d r}{r}+(1 / 2 \pi) \int_{B(x, 1)} f(y) m(d y) .
$$

A dominated convergence argument shows that $k_{1} f$ is continuous on $\mathbb{R}^{2}$. As for $k_{2}$, we have

$$
\chi_{B(x, 1)^{c}}(y) \log |x-y| \leq \tau+|y| \quad\left(y \in \mathbb{R}^{2}\right)
$$

for all $x \in B(0, \tau)(\tau>0)$. Continuity of $k_{2} f$ follows by another appeal to dominated convergence.

(ii) Write

$$
r^{\lambda}=g^{\lambda}-k+\left\{r\left(\lambda^{1 / 2}\right)-g^{\lambda}(u)\right\}\langle\cdot, 1\rangle 1 .
$$

Now $\mathscr{H}_{s} \subseteq L^{p}\left(\mathbb{R}^{2}\right)$ for each $p>1$. By the Sobolev embedding [2] Theorem 1.2.4, $g^{\lambda}: \mathscr{H}_{s} \rightarrow C\left(\mathbb{R}^{2}\right)$. This and (i) establish the claim.

(iii) Pick $0<\alpha<1 / 3$. For each $x \in \mathbb{R}^{2}$ introduce sets

$$
\begin{aligned}
& A_{1}^{\lambda}:=\left\{y \in \mathbb{R}^{2}:|y-x| \leq 1\right\}, \\
& A_{2}^{\lambda}:=\left\{y \in \mathbb{R}^{2}: 1<|y-x| \leq \lambda^{-\alpha}\right\}, \\
& A_{3}^{\lambda}:=\left\{y \in \mathbb{R}^{2}: \lambda^{-\alpha}<|y-x| \leq \delta \lambda^{-1 / 2}\right\}, \\
& A_{4}^{\lambda}:=\left\{y \in \mathbb{R}^{2}:|y-x|>\delta \lambda^{-1 / 2}\right\},
\end{aligned}
$$

for $\lambda$ sufficiently small (where the $x$-dependence has been suppressed for the sake of legibility). Define

$$
r_{j}^{\lambda} f(x):=\int_{A_{j}^{\lambda}} r\left(\lambda^{1 / 2}|x-y|\right) f(y) m(d y) \quad\left(x \in \mathbb{R}^{2}\right) \quad(j=1,2,3,4) .
$$


Fix $\tau>0$. For $0<\lambda<\delta^{2}$,

$$
\left|r_{1}^{\lambda} f(x)\right| \leq(C / 4) \lambda\left\{\int_{|x-y| \leq 1}\left(\log \lambda^{1 / 2}|x-y|\right)^{2} m(d y)\right\}^{1 / 2}\|f\|_{\mathscr{H}_{s}}
$$

by (3.2). In particular, $g^{\lambda}(u) r_{1}^{\lambda} f \rightarrow 0$ uniformly on $B(0, \tau)$ as $\lambda \downarrow 0$.

Again from (3.2), for $\lambda>0$ small,

$$
\left|r_{2}^{\lambda} f(x)\right| \leq(C / 4) \lambda\left\{\int_{1<|x-y| \leq \lambda^{-\alpha}}|x-y|^{4}\left[(1 / 2)(\log \lambda)^{2}+2(\log |x-y|)^{2}\right] m(d y)\right\}^{1 / 2}\|f\|_{\mathscr{H}_{s}} .
$$

Choose $0<\eta<1 / \alpha-3$. An estimate of the form $|\log | x-y|| \leq c_{\eta}|x-y|^{\eta}$ holds on $A_{2}^{\lambda}$. Also,

$$
\left\{\int_{1<|x-y| \leq \lambda^{-\alpha}}|x-y|^{4+2 \eta} m(d y)\right\}^{1 / 2} \leq\left\{\frac{\pi}{3+\eta}\right\}^{1 / 2} \lambda^{-\alpha(3+\eta)}
$$

So $g^{\lambda}(u) r_{2}^{\lambda} f \rightarrow 0$ uniformly on $B(0, \tau)$ as $\lambda \downarrow 0$.

The kernel $r\left(\lambda^{1 / 2}|x-y|\right)$ is bounded by a constant $c^{\prime}$ (say) on $A_{3}^{\lambda}$. Thus, for any $x \in B(0, \tau)$,

$$
\begin{aligned}
\left|r_{3}^{\lambda} f(x)\right| & \leq c^{\prime} \int_{\lambda^{-\alpha}<|x-y| \leq \delta \lambda^{-1 / 2}}|f(y)| m(d y) \\
& \leq c^{\prime} \int_{\lambda^{-\alpha}<|x-y|}|f(y)| m(d y) \\
& \leq c^{\prime} \int_{B\left(0, \lambda^{-\alpha}-\tau\right)^{c}}|f(y)| m(d y) \\
& \leq c^{\prime}\|f\|_{\mathscr{H}_{s}}\left\{\int_{B\left(0, \lambda^{-\alpha}-\tau\right)^{c}}\langle y\rangle^{-2 s} m(d y)\right\}^{1 / 2}
\end{aligned}
$$

provided $\lambda$ is sufficiently small. The weight function $\langle\cdot\rangle^{-2 s}$ is integrable because $s>1$. Thus $g^{\lambda}(u) r_{3}^{\lambda} f \rightarrow 0$ uniformly on $B(0, \tau)$ as $\lambda \downarrow 0$.

By (3.4),

$$
r_{4}^{\lambda} f(x)=\int_{|x-y|>\delta \lambda^{-1 / 2}}\left\{g^{\lambda}(x-y)-a_{0}+\imath \pi c_{0}+c_{0} \log \lambda-k(x-y)\right\} f(y) m(d y) .
$$

An estimate of the form (9.2) gives

$$
\begin{aligned}
\left|\int_{|x-y|>\delta \lambda^{-1 / 2}} g^{\lambda}(x-y) f(y) m(d y)\right| & \leq(c / 4) \lambda^{-1 / 4} \int_{|x-y|>\delta \lambda^{-1 / 2}}|x-y|^{-1 / 2}|f(y)| m(d y) \\
& \leq(c / 4) \delta^{-1 / 2} \lambda^{1 / 2} \int_{|x-y|>\delta \lambda^{-1 / 2}}|f(y)| m(d y) .
\end{aligned}
$$

Uniform convergence can be derived in a way similar to the treatment of $r_{3}^{\lambda}$.

Choose $\eta>0$ such that $s-\eta>1$. For $x \in B(0, \tau)$ and $\lambda$ small,

$$
\begin{aligned}
\left|\int_{|x-y|>\delta \lambda^{-1 / 2}} k(x-y) f(y) m(d y)\right| & \leq c_{\eta} \int_{|x-y|>\delta \lambda^{-1 / 2}}|x-y|^{\eta}|f(y)| m(d y) \\
& \leq c_{\eta}^{\prime} \int_{|x-y|>\delta \lambda^{-1 / 2}}\langle y\rangle^{\eta}|f(y)| m(d y) \\
& \leq c_{\eta}^{\prime} \int_{B\left(0, \delta \lambda^{-1 / 2}-\tau\right)^{c}}\langle y\rangle^{\eta}|f(y)| m(d y) \\
& \leq c_{\eta}^{\prime}\|f\|_{\mathscr{H}_{s}}\left\{\int_{B\left(0, \delta \lambda^{-1 / 2}-\tau\right)^{c}}\langle y\rangle^{-2(s-\eta)} m(d y)\right\}^{1 / 2}
\end{aligned}
$$


for appropriate constants $c_{\eta}, c_{\eta}^{\prime}$. The remaining terms in $r_{4}^{\lambda}$ can be dealt with using similar analysis. Consequently, $g^{\lambda}(u) r_{4}^{\lambda} f \rightarrow 0$ uniformly on $B(0, \tau)$ as $\lambda \downarrow 0$.

Let $M=\left(\Omega, \mathscr{M}, X_{t}, \mathbb{P}_{x}\right)$ be Brownian motion on $\mathbb{R}^{2}$ with transition function $p(t, \cdot)$ given by

$$
p(t, x)=(4 \pi t)^{-1} e^{-|x|^{2} / 4 t} \quad(t>0)
$$

(see [20] for example). Put $\sigma_{K}:=\inf \left\{t>0: X_{t} \in K\right\}$, the first hitting time of $K$. The hitting operators $h_{K}^{\lambda}$ are defined by

$$
h_{K}^{\lambda} f:=\mathbb{E} .\left[e^{-\lambda \sigma_{K}} f\left(X_{\sigma_{K}}\right): \sigma_{K}<\infty\right]
$$

for measurable $f \geq 0$. If $\lambda=0$, we write $h_{K}$ instead of $h_{K}^{0}$. The $\lambda$-potential of $K$ is the function

$$
p_{K}^{\lambda}:=h_{K}^{\lambda} 1 .
$$

In case $\lambda=0, p_{K}:=p_{K}^{0} \equiv 1$ by recurrence ([20] Proposition 2.9, for example). Set

$$
W_{K}^{\lambda}:=g^{\lambda}(u)\left\{1-p_{K}^{\lambda}\right\} .
$$

In case $K$ is nonpolar, the limit

$$
W_{K}:=\lim _{\lambda \downarrow 0} W_{K}^{\lambda}
$$

exists finitely according to 20 Theorem 3.4.2. The equilibrium measure $\mu_{K}$ is the unique probability measure concentrated on $K^{r}$ whose potential $k \mu_{K}$ is constant on $K^{r}$. Here, $K^{r}$ denotes the set of regular points for $K$ ([20] 2.3). This constant value is the Robin constant $R(K)$ of $K$. We use the notation

$$
C(K):=-4 \pi R(K) .
$$

From [20] Theorem 3.4.12,

$$
k \mu_{K}=R(K)-W_{K} .
$$

In particular, $W_{K} \in \mathscr{H}_{-s}$ for any $s>1$. The 1 -capacity of $K$ is denoted $C_{1}(K)$ (see [10]). It holds that

$$
C_{1}(K)=\left\langle 1, p_{K}^{1}\right\rangle
$$

We use the notation $g_{K}^{\lambda}(\lambda>0)$ to refer to the $\lambda$-potential operator with kernel

$$
g_{K}^{\lambda}(x, y):=\int_{0}^{\infty} e^{-\lambda t} q_{K}(t, x, y) d t \quad\left(x, y \in \mathbb{R}^{2}\right) ;
$$

to clarify, $q_{K}(t, x, y)=p(t, x-y)-r_{K}(t, x, y)$ with

$$
r_{K}(t, x, y):=\mathbb{E}_{x}\left[p\left(t-\sigma_{K}, X\left(\sigma_{K}\right)-y\right): \sigma_{K}<t\right]
$$

as in 20] 2.5. We have that $g_{K}^{\lambda} f=R_{Y}(-\lambda) f m$-a.e. on $Y$ for $f \in L^{2}(Y)$. If $\lambda=0$ the notation $g_{K}$ is sometimes used.

As in [20] 3.4, the fundamental identities for logarithmic potentials read

$$
\begin{aligned}
& k^{\lambda}=g_{K}^{\lambda}+h_{K}^{\lambda} k^{\lambda}-\langle\cdot, 1\rangle W_{K}^{\lambda}, \\
& k=g_{K}+h_{K} k-\langle\cdot, 1\rangle W_{K} .
\end{aligned}
$$


Lemma 3.1. For any $\lambda, \mu \geq 0$ with $\lambda+\mu>0$ we have that

$$
g_{K}^{\lambda} p_{K}^{\mu}=-(\mu-\lambda)^{-1}\left\{p_{K}^{\mu}-p_{K}^{\lambda}\right\} .
$$

Proof. The result follows as in the proof of [18] Proposition 4.13.

Let $T_{r}:=\inf \left\{t>0: X_{t} \notin B(0, r)\right\}$ stand for the first exit time of $B(0, r)$. The notation $B(0, r)$ signifies the open ball with centre at the origin and radius $r>0$.

Lemma 3.2. For each $r>0$,

$$
\mathbb{E}_{0}\left[e^{-T_{r}}\right]=1 / I_{0}(r)
$$

where $I_{0}$ stands for the modified Bessel function of order zero. Moreover,

$$
\mathbb{E}_{0}\left[e^{-T_{r}}\right] \sim(2 \pi r)^{1 / 2} e^{-r}
$$

as $r \rightarrow \infty$.

Proof. A direct computation leads to the identity. The asymptotic behaviour follows as in [25] 3.6.

Proposition 3.1. We have that

$$
\left\langle W_{K}, p_{K}^{1}\right\rangle=1
$$

Proof. In a similar way to the proof of [20] Proposition 3.4.4, we write

$$
\left\langle k(\cdot, y)-k(0, y) 1, p_{K}^{1}\right\rangle=g_{K} p_{K}^{1}(y)+\left\langle h_{K}[k(\cdot, y)-k(0, y) 1], p_{K}^{1}\right\rangle-\left\langle W_{K}, p_{K}^{1}\right\rangle \quad\left(y \in \mathbb{R}^{2}\right)
$$

The function

$$
k(x, y)-k(0, y)=-\frac{1}{2 \pi} \log \frac{|x-y|}{|y|} \quad\left(x \in \mathbb{R}^{2} \backslash\{y\}\right)
$$

converges to zero uniformly on compacts as $y \rightarrow \infty$. From Lemma 3.1

$$
g_{K} p_{K}^{1}(y)=1-p_{K}^{1}(y)
$$

This latter converges to 1 in the limit $y \rightarrow \infty$ by Lemma 3.2 . Therefore, the expression on the right-hand side of (3.13) converges to $1-\left\langle W_{K}, p_{K}^{1}\right\rangle$.

We now show that the left-hand side vanishes in the limit. Let $\varepsilon>0$ and choose $0<\delta<1$ with the property that

$$
|\log (1+\tau)|<2 \pi \varepsilon \text { for any } \tau \in \mathbb{R} \text { with }|\tau|<\delta .
$$

For any $x, y \in \mathbb{R}^{2}$ with $|x|<\delta|y|$ we have

$$
|k(x, y)-k(0, y)|<\varepsilon
$$

The left-hand side in (3.13) may be written,

$$
\left\langle k(\cdot, y)-k(0, y) 1, p_{K}^{1}\right\rangle=\int_{\mathbb{R}^{2}}\{k(x, y)-k(0, y)\} p_{K}^{1}(x) m(d x) .
$$

Decompose $\mathbb{R}^{2}$ into the disjoint union $\mathbb{R}^{2}=F_{y} \dot{\cup} G_{y}$ with

$$
F_{y}:=\left\{x \in \mathbb{R}^{2}:|x|<\delta|y|\right\} \text { and } G_{y}:=\left\{x \in \mathbb{R}^{2}:|x| \geq \delta|y|\right\} .
$$


The integral over $F_{y}$ is bounded by $\varepsilon \operatorname{Cap}_{1}(K)$ in modulus by (3.14). Re-write the integral over $G_{y}$ as

$$
-k(0, y) \int_{G_{y}} p_{K}^{1}(x) m(d x)+\int_{A_{y}} k(x, y) p_{K}^{1}(x) m(d x)+\int_{B_{y}} k(x, y) p_{K}^{1}(x) m(d x)
$$

where

$$
A_{y}:=\left\{x \in \mathbb{R}^{2}:|x| \geq \delta|y|,|x-y| \leq 1\right\} \text { and } B_{y}:=\left\{x \in \mathbb{R}^{2}:|x| \geq \delta|y|,|x-y|>1\right\} .
$$

The first two integrals vanish in the limit by Lemma 3.2 for the last, use in addition the estimate

$$
|\log | x-y|| \leq|x|+|y| \text { for }|x-y|>1 \text {. }
$$

Lemma 3.3. Let $s>1$. Then

(i) $W_{K}^{\lambda} \rightarrow W_{K}$ in $\mathscr{H}_{-s}$ as $\lambda \downarrow 0$;

(ii) $R_{Y}(-\lambda) \rightarrow R_{Y}(0)$ strongly in $B\left(\mathscr{H}_{s}, \mathscr{H}_{-s}\right)$ as $\lambda \downarrow 0$.

Proof. From (3.11), (3.12) and Lemma 3.1 we have

$$
\begin{aligned}
k^{\lambda} p_{K}^{1} & =\frac{1}{1-\lambda}\left\{p_{K}^{\lambda}-p_{K}^{1}\right\}+h_{K}^{\lambda} k^{\lambda} p_{K}^{1}-C_{1}(K) W_{K}^{\lambda}, \\
k p_{K}^{1} & =1-p_{K}^{1}+h_{K} k p_{K}^{1}-C_{1}(K) W_{K} .
\end{aligned}
$$

Consequently,

$\left[k^{\lambda}-k\right] p_{K}^{1}=\frac{1}{1-\lambda}\left\{p_{K}^{\lambda}-1\right\}+\frac{\lambda}{1-\lambda}-\frac{\lambda}{1-\lambda} p_{K}^{1}+h_{K}^{\lambda}\left[k^{\lambda}-k\right] p_{K}^{1}+\left(h_{K}^{\lambda}-h_{K}\right) k p_{K}^{1}-C_{1}(K)\left\{W_{K}^{\lambda}-W_{K}\right\}$.

(i) follows with the help of Corollary 3.1 and the dominated convergence theorem.

From (3.11) resp. (3.12) it can be seen that $g_{K}^{\lambda}$ resp. $g_{K}$ map $\mathscr{H}_{s}$ boundedly into $\mathscr{H}_{-s}$ for any $s>1$. Further,

$$
g_{K}^{\lambda}-g_{K}=k^{\lambda}-k+h_{K}^{\lambda}\left(k^{\lambda}-k\right)+\left(h_{K}^{\lambda}-h_{K}\right) k-\langle\cdot, 1\rangle\left\{W_{K}^{\lambda}-W_{K}\right\} .
$$

The claim in (ii) now follows from Corollary 3.1 Theorem 3.1 and (i) above. We use the relation (3.6).

Proposition 3.2. It holds that

$$
\lim _{\lambda \downarrow 0} g^{\lambda}(u)\left\{1-\left\langle W_{K}^{\lambda}, p_{K}^{1}\right\rangle\right\}=R(K) .
$$

Proof. Applying (3.11) to the equilibrium measure $\mu_{K}$ we obtain the identity

$$
k^{\lambda} \mu_{K}=h_{K}^{\lambda} k^{\lambda} \mu_{K}-W_{K}^{\lambda}
$$

with the help of [20] Theorem 4.4.3 as $\mu_{K}$ is a probability measure with support in $K^{r}$. We derive

$$
\left\langle W_{K}^{\lambda}, p_{K}^{1}\right\rangle=\left\langle h_{K}^{\lambda} k^{\lambda} \mu_{K}, p_{K}^{1}\right\rangle-\left\langle k^{\lambda} \mu_{K}, p_{K}^{1}\right\rangle .
$$

In virtue of (3.9) we have

$$
\left\langle W_{K}, p_{K}^{1}\right\rangle=R(K)\left\langle 1, p_{K}^{1}\right\rangle-\left\langle k \mu_{K}, p_{K}^{1}\right\rangle .
$$

Using Proposition 3.1 we proceed,

$$
\begin{aligned}
1-\left\langle W_{K}^{\lambda}, p_{K}^{1}\right\rangle & =R(K)\left\langle 1, p_{K}^{1}\right\rangle-\left\langle h_{K}^{\lambda}\left[k^{\lambda}-k\right] \mu_{K}, p_{K}^{1}\right\rangle-\left\langle h_{K}^{\lambda} k \mu_{K}, p_{K}^{1}\right\rangle+\left\langle\left[k^{\lambda}-k\right] \mu_{K}, p_{K}^{1}\right\rangle \\
& =\frac{R(K)}{g^{\lambda}(u)}\left\langle W_{K}^{\lambda}, p_{K}^{1}\right\rangle-\left\langle h_{K}^{\lambda}\left[k^{\lambda}-k\right] \mu_{K}, p_{K}^{1}\right\rangle+\left\langle\left[k^{\lambda}-k\right] \mu_{K}, p_{K}^{1}\right\rangle .
\end{aligned}
$$

The result now follows from Lemma 3.3 (i), (3.6), (3.2), Theorem 3.1 (iii) and duality. 


\section{Construction of inverse operators}

Let $X$ be a complex Banach space with dual space $X^{\prime}$ and duality pairing $\langle\cdot, \cdot \cdot$. Let $B(X)$ stand for the collection of bounded linear operators on $X$. The notation $A^{\times}$stands for the adjoint operator of $A \in B(X)$.

Lemma 4.1. Assume that $A \in B(X)$ is bijective with inverse $B$. Let $y \in X, f \in X^{\prime}$ and $\sigma \in \mathbb{C}$. Define $A_{\sigma}$ to be the rank-one perturbation of $A$ given by

$$
A_{\sigma}:=A+\sigma\langle\cdot, f\rangle y .
$$

Suppose that

$$
\alpha:=1+\sigma\langle B y, f\rangle \neq 0 .
$$

Then $A_{\sigma}$ is bijective and has inverse given by

$$
B_{\sigma}=B-\alpha^{-1} \sigma\left\langle\cdot, B^{\times} f\right\rangle B y .
$$

Proof. Verify by direct computation that $A_{\sigma} B_{\sigma}=B_{\sigma} A_{\sigma}=I$.

Lemma 4.2. Let $\delta>0$. Suppose that $\left(A_{\lambda}\right)_{\lambda \in(0, \delta)}$ is a family of operators in $B(X)$. Assume that

(i) $A_{\lambda} \rightarrow A$ strongly as $\lambda \downarrow 0$ for some $A \in B(X)$;

(ii) for each $\lambda \in(0, \delta), A_{\lambda}$ is bijective with inverse $B_{\lambda}$;

(iii) $B_{\lambda} \rightarrow B$ strongly as $\lambda \downarrow 0$ for some $B \in B(X)$.

Then $A$ is bijective and has inverse $B$.

Proof. Given $x \in X$ write, for example,

$$
B A x-x=\left(B-B_{\lambda}\right) A x+B_{\lambda}\left(A-A_{\lambda}\right) x .
$$

By the uniform boundedness principle there exists a finite constant $c$ such that $\left\|B_{\lambda}\right\| \leq c<\infty$ for all $\lambda \in(0, \delta)$. Take limits on the right-hand side using (i) and (iii) to see that $B A x-x=0$.

Lemma 4.3. For any $\lambda>0$,

(i) $\left[I-R_{Y}^{(-1)}\left((1-\lambda)^{-1}\right) V\right] 1=g^{\lambda}(u)^{-1} W_{K}^{\lambda}+\lambda p_{K}^{\lambda}$;

(ii) $\left[I-V R_{Y}^{(-1)}\left((1-\lambda)^{-1}\right)\right] p_{K}^{1}=-V\left[g^{\lambda}(u)^{-1} W_{K}^{\lambda}+\lambda p_{K}^{\lambda}\right]$.

Proof. Item (ii) follows from (i) via the identity $p_{K}^{1}=-V 1$. This last follows from Lemma 3.1 Again by this lemma,

$$
\begin{aligned}
{\left[I-R_{Y}^{(-1)}\left((1-\lambda)^{-1}\right) V\right] 1 } & =1+R_{Y}^{(-1)}\left((1-\lambda)^{-1}\right) p_{K}^{1} \\
& =1-(1-\lambda)\left\{I+(1-\lambda) R_{Y}(-\lambda)\right\} p_{K}^{1} \\
& =1-(1-\lambda) p_{K}^{1}-(1-\lambda)^{2} g_{K}^{\lambda} p_{K}^{1} \\
& =1-(1-\lambda) p_{K}^{1}+(1-\lambda)\left\{p_{K}^{1}-p_{K}^{\lambda}\right\} \\
& =g^{\lambda}(u)^{-1} W_{K}^{\lambda}+\lambda p_{K}^{\lambda} .
\end{aligned}
$$

Define

$$
\begin{aligned}
& A \quad:=I-[I+k] V, \\
& A_{\lambda}:=I+R^{(-1)}\left((1-\lambda)^{-1}\right) V-g^{\lambda}(u)\left\langle\cdot, p_{K}^{1}\right\rangle 1 \quad(\lambda>0) .
\end{aligned}
$$


Proposition 4.1. Let $s>1$. Then

(i) $A \in B\left(\mathscr{H}_{-s}\right)$;

(ii) $A_{\lambda} \in B\left(\mathscr{H}_{-s}\right)$ for any $\lambda>0$;

(iii) $A_{\lambda} \rightarrow A$ strongly in $B\left(\mathscr{H}_{-s}\right)$ as $\lambda \downarrow 0$;

(iv) $A W_{K}=R(K) 1$.

(v) $A^{\times} V W_{K}=-R(K) p_{K}^{1}$.

Proof. Statement (i) flows from [18] Theorem 2.1 and Lemma 3.1. From Theorem 2.1 and [18] Lemma $3.1, R(-\lambda) \in B\left(\mathscr{H}_{s}, \mathscr{H}_{-s}\right)$; (ii) now follows. For (iii), we may write

$$
A_{\lambda}=I-(1-\lambda)\left[I+(1-\lambda) k^{\lambda}\right] V+\lambda(\lambda-2) g^{\lambda}(u)\left\langle\cdot, p_{K}^{1}\right\rangle 1
$$

with the help of (3.5). Thus,

$$
A_{\lambda}-A=\lambda V+\left[k-k^{\lambda}\right] V+\lambda(2-\lambda)\left\{k^{\lambda} V-g^{\lambda}(u)\left\langle\cdot, p_{K}^{1}\right\rangle 1\right\} .
$$

The strong convergence follows from Corollary 3.1

As for the identity (iv), from Lemma 3.1 and the first resolvent identity, we derive

$$
\begin{array}{ll}
V p_{K}^{\lambda} & =(1-\lambda)^{-1}\left\{p_{K}^{\lambda}-p_{K}^{1}\right\}-R(-1) p_{K}^{\lambda}, \\
R(-\lambda) V p_{K}^{\lambda} & =(1-\lambda)^{-1}\left\{R(-1) p_{K}^{\lambda}-R(-\lambda) p_{K}^{1}\right\} ;
\end{array}
$$

the second flowing from the first. With their help, a computation leads to the identity

$$
A_{\lambda} W_{K}^{\lambda}=g^{\lambda}(u)\left\{1-\left\langle W_{K}^{\lambda}, p_{K}^{1}\right\rangle\right\} 1-\lambda g^{\lambda}(u) p_{K}^{1}+\lambda(\lambda-1) g^{\lambda}(u) R(-\lambda) p_{K}^{1} .
$$

By (3.5) and (3.10),

$$
\lambda(\lambda-1) g^{\lambda}(u) R(-\lambda) p_{K}^{1}=\lambda(\lambda-1) g^{\lambda}(u)\left\{k^{\lambda} p_{K}^{1}+g^{\lambda}(u) C_{1}(K) 1\right\} \rightarrow 0 \text { in } \mathscr{H}_{-s} \text { as } \lambda \downarrow 0
$$

by Corollary 3.1. This shows that

$$
A_{\lambda} W_{K}^{\lambda} \rightarrow R(K) 1 \text { in } \mathscr{H}_{-s} \text { as } \lambda \downarrow 0
$$

by Proposition 3.2 . Finally, write

$$
A W_{K}-R(K) 1=\left(A-A_{\lambda}\right) W_{K}+A_{\lambda}\left(W_{K}-W_{K}^{\lambda}\right)+A_{\lambda} W_{K}^{\lambda}-R(K) 1
$$

and use (4.3), Lemma 3.3 (i), and (iii). Of course, the family $\left(A_{\lambda}\right)_{\lambda \in(0,1)}$ is bounded by the uniform boundedness principle.

Lastly, $A^{\times} V W_{K}=V A W_{K}=-R(K) p_{K}^{1}$ by (iv).

Lemma 4.4. Let $s>1$. Assume that $R(K) \neq 0$. Then there exists $\delta>0$ such that for each $\lambda \in(0, \delta)$ the operator $A_{\lambda}$ is bijective with inverse $B_{\lambda}$ given by

$$
B_{\lambda}=I-R_{Y}^{(-1)}\left((1-\lambda)^{-1}\right) V-\frac{1}{g^{\lambda}(u) \alpha_{\lambda}}\left\langle\cdot, V\left[W_{K}^{\lambda}+\lambda g^{\lambda}(u) p_{K}^{\lambda}\right]\right\rangle\left(W_{K}^{\lambda}+\lambda g^{\lambda}(u) p_{K}^{\lambda}\right)
$$

where

$$
\alpha_{\lambda}=1-\left\langle W_{K}^{\lambda}, p_{K}^{1}\right\rangle+\lambda g^{\lambda}(u)\left\langle p_{K}^{\lambda}, p_{K}^{1}\right\rangle .
$$


Proof. The counterpart $\alpha_{\lambda}$ of (4.1) reads

$$
\alpha_{\lambda}:=1-g^{\lambda}(u)\left\langle\left[I-R_{Y}^{(-1)}\left((1-\lambda)^{-1}\right) V\right] 1, p_{K}^{1}\right\rangle=1-\left\langle W_{K}^{\lambda}, p_{K}^{1}\right\rangle+\lambda g^{\lambda}(u)\left\langle p_{K}^{\lambda}, p_{K}^{1}\right\rangle
$$

after simplification using Lemma 4.3. By Proposition 3.2 $g^{\lambda}(u) \alpha_{\lambda} \rightarrow R(K)$ as $\lambda \downarrow 0$. Consequently, there exists $\delta>0$ such that $\alpha_{\lambda} \neq 0$ for $\lambda \in(0, \delta)$. By Lemma 4.1, $A_{\lambda}$ is bijective with inverse as in (4.4); again, after making use of Lemma 4.3.

Define

$$
B_{0}:=I+\left[I+R_{Y}(0)\right] V .
$$

Lemma 4.5. The following identities hold:

(i) $B_{0} 1=0$;

(ii) $B_{0}^{\times} p_{K}^{1}=0$.

Proof. By Lemma 3.1 .

$$
B_{0} 1=1+\left[I+R_{Y}(0)\right] V 1=1-\left[I+R_{Y}(0)\right] p_{K}^{1}=1-p_{K}^{1}-\left\{1-p_{K}^{1}\right\}=0
$$

giving (i). For (ii), $B_{0}^{\times} p_{K}^{1}=-B_{0}^{\times} V 1=-V B_{0} 1=0$.

In case $R(K) \neq 0$, define

$$
B:=B_{0}-R(K)^{-1}\left\langle\cdot, V W_{K}\right\rangle W_{K} .
$$

Proposition 4.2. Let $s>1$. Assume that $R(K) \neq 0$. Then

(i) $B_{\lambda} \rightarrow B$ strongly in $B\left(\mathscr{H}_{-s}\right)$ as $\lambda \downarrow 0$;

(ii) $A$ is bijective in $B\left(\mathscr{H}_{-s}\right)$ with inverse $B$ as in 4.5);

(iii) $B 1=R(K)^{-1} W_{K}$;

(iv) $B^{\times}=I+V\left[I+R_{Y}(0)\right]-R(K)^{-1}\left\langle\cdot, W_{K}\right\rangle V W_{K}$;

(v) $B^{\times} p_{K}^{1}=-R(K)^{-1} V W_{K}$.

Proof. (i) follows from Proposition 3.2 and Lemma 3.3. This together with Lemmas 4.2 and 4.4 , and Proposition 4.1 (iii) yield (ii). To see (iii) use Proposition 4.1 (iv). For (v) use the identity $B^{\times} V=V B$ and (iii).

Lemma 4.6. Let $s>1$. Set

$$
\begin{aligned}
\mathscr{M} & :=\left\{u \in \mathscr{H}_{-s}:\left\langle u, p_{K}^{1}\right\rangle=0\right\}, \\
\mathscr{W} & :=\left\{u \in \mathscr{H}_{-s}:\left\langle u, V W_{K}\right\rangle=0\right\} .
\end{aligned}
$$

Then

(i) $B_{0} A=I$ on $\mathscr{M}$;

(ii) $A B_{0}=I$ on $\mathscr{W}$.

Proof. Note that $A_{\lambda}=I+R^{(-1)}\left((1-\lambda)^{-1}\right) V$ on $\mathscr{M}$ for each $\lambda>0$. Set

$$
B_{0, \lambda}:=I-R_{Y}^{(-1)}\left((1-\lambda)^{-1}\right) V
$$

on $\mathscr{H}_{-s}$. Then $B_{0, \lambda} \rightarrow B_{0}$ strongly in $B\left(\mathscr{H}_{-s}\right)$ as $\lambda \downarrow 0$. By the second resolvent identity and density of $\mathscr{H} \cap \mathscr{M}$ in $\mathscr{M}, B_{0, \lambda} A_{\lambda}=I$ on $\mathscr{M}$. By Proposition 4.1 (iii) and as in Lemma 4.2 we obtain $B_{0} A=I$ on $\mathscr{M}$. This establishes (i).

From Lemma 4.3 (ii), $B_{0, \lambda}^{\times} p_{K}^{1}=-g^{\lambda}(u)^{-1} V W_{K}^{\lambda}-\lambda V p_{K}^{\lambda}$. Hence,

$$
g^{\lambda}(u)\left\langle B_{0, \lambda} u, p_{K}^{1}\right\rangle=\left\langle u,-V W_{K}^{\lambda}-\lambda g^{\lambda}(u) V p_{K}^{\lambda}\right\rangle \rightarrow-\left\langle u, V W_{K}\right\rangle=0 \text { as } \lambda \downarrow 0
$$

for $u \in \mathscr{W}$. Therefore, $A_{\lambda} B_{0, \lambda} u=u-g^{\lambda}(u)\left\langle B_{0, \lambda} u, p_{K}^{1}\right\rangle \rightarrow u$ as $\lambda \downarrow 0$. Now use strong convergence to obtain (ii). 


\section{A lattice-point counting lemma}

We require a simple lattice-point counting lemma. Let us make the following definitions. For $n \in \mathbb{N}$ and $\mathbb{Z} \ni k<0$ set

$$
\begin{aligned}
A(n, k) & :=\left\{x \in \mathbb{Z}^{n}: x_{j} \leq 1 \text { for } j=1,2, \ldots, n \text { and } \sum_{j=1}^{n} x_{j}=k\right\} \\
a(n, k) & :=\operatorname{Card}(A(n, k)) .
\end{aligned}
$$

Lemma 5.1. For $n \in \mathbb{N}$ and $\mathbb{Z} \ni k<0$, it holds that

$$
a(n, k) \leq a(n)\{|k|+(3 / 2) n\}^{n-1} .
$$

The constant $a(n)$ is given by

$$
a(n)=\frac{\sqrt{n}}{\alpha(n-1)} \frac{2^{n-1}}{(n-1) !} .
$$

Here, $\alpha(n)$ stands for the volume of the unit ball $B(0,1)$ in $\mathbb{R}^{n}$; it is understood that $\alpha(0)=1$.

Proof. First, notice that $a(1, k)=1$. For $n=2,3, \ldots$ and $r \leq(3 / 2) n$ set

$$
\begin{aligned}
H(n, r) & :=\left\{x \in \mathbb{R}^{n}: x_{j} \leq 3 / 2 \text { for } j=1,2, \ldots, n \text { and } \sum_{j=1}^{n} x_{j}=r\right\}, \\
h(n, r) & :=\sigma(H(n, r)),
\end{aligned}
$$

where $\sigma$ stands for surface area measure. We claim that

$$
h(n, r)=\frac{\sqrt{n}}{(n-1) !}\{|r|+(3 / 2) n\}^{n-1}
$$

for $r<0$. To see this, introduce the set

$$
S(n, r):=\left\{x \in \mathbb{R}^{n}: x_{j} \geq 0 \text { for } j=1,2, \ldots, n \text { and } \sum_{j=1}^{n} x_{j}=r\right\}
$$

for $r \geq 0$ and $n \geq 2$. As in [27] (for example) its surface area is given by

$$
s(n, r):=\sigma(S(n, r))=\sqrt{n} r^{n-1} /(n-1) ! .
$$

Since

$$
H(n, r)=(3 / 2)(1, \ldots, 1)-S(n,-r+(3 / 2) n),
$$

the formula (5.1) follows.

To prove the lemma, note the inclusion

$$
\bigcup_{x \in A(n, k)} B(x, 1 / 2) \cap H(n, k) \subseteq H(n, k)
$$

where the left-hand side is a disjoint union. Computing surface area using (5.1) yields the claim. 


\section{Asymptotics of the spectral shift function}

The result below follows from Theorem 2.1 the method of proof is similar to that used in the proof of [18] Lemma 4.7.

Lemma 6.1. Let $l \in \mathbb{N}_{0}$ and $s>2 l+1$. Then for $\zeta \in \mathbb{C} \backslash[0, \infty)$,

$$
\left\|R^{(-1)}\left((1+\zeta)^{-1}\right)-\sum_{j=0}^{l} \sum_{k=0}^{1} \zeta^{j} \eta^{k} A_{j}^{k}\right\|_{B\left(\mathscr{H}_{s}, \mathscr{H}_{-s}\right)}=o\left(|\zeta|^{l}\right)
$$

as $\zeta \rightarrow 0$. The coefficients are given by

$$
\begin{aligned}
& A_{0}^{1}=-K_{0}^{1}, \\
& A_{0}^{0}=-I-K_{0}^{0}, \\
& A_{1}^{1}=-2 K_{0}^{1}-K_{1}^{1}, \\
& A_{1}^{0}=-I-2 K_{0}^{0}-K_{1}^{0}, \\
& A_{j}^{k}=-K_{j}^{k}-2 K_{j-1}^{k}-K_{j-2}^{k} \text { for } j \geq 2 \text { and } k \in\{0,1\} .
\end{aligned}
$$

In the context of the last Lemma, we may write

$$
I+R^{(-1)}\left((1+\zeta)^{-1}\right) V=I-[I+k] V+\sigma\left\langle\cdot, p_{K}^{1}\right\rangle 1+\sum_{j=1}^{l} \sum_{k=0}^{1} \zeta^{j} \eta^{k} A_{j}^{k} V+o\left(|\zeta|^{l}\right)
$$

in $B\left(\mathscr{H}_{-s}\right)$ as $\mathbb{C} \backslash[0, \infty) \ni \zeta \rightarrow 0$. We use the shorthand

$$
\sigma=a+b \eta
$$

where $a=a_{0}$ and $b=c_{0}$. Define

$$
A_{\sigma}:=A+\sigma\left\langle\cdot, p_{K}^{1}\right\rangle 1
$$

with $A$ as in (4.2).

Proposition 6.1. Let $s>1$. Then for small $\zeta \in \mathbb{C} \backslash[0, \infty), A_{\sigma} \in B\left(\mathscr{H}_{-s}\right)$ is bijective with inverse given by

$$
B_{\sigma}:=B_{0}+\sum_{k=-\infty}^{-1} \theta_{k} \eta^{k}\left\langle\cdot V W_{K}\right\rangle W_{K}
$$

where

$$
\theta_{k}:=(-1)^{-k}(1 / b)\left(\frac{R(K)+a}{b}\right)^{-(k+1)} \text { for } k=-1,-2, \ldots
$$

Moreover, there exists a finite constant c such that

$$
\left\|B_{\sigma}\right\|_{B\left(\mathscr{H}_{-s}\right)} \leq c<\infty
$$

for small $\zeta \in \mathbb{C} \backslash[0, \infty)$.

For later use, we introduce the quantity

$$
\theta:=\left|\frac{R(K)+a}{b}\right|
$$


Note that $\theta$ is invertible; in fact, $\theta \geq \pi$ for all values of $R(K) \in \mathbb{R}$.

Proof. We first treat the case $R:=R(K) \neq 0$. By Lemma 4.2 (iii) and Proposition 3.1.

$$
\alpha_{\sigma}:=1+\sigma\left\langle B 1, p_{K}^{1}\right\rangle=1+\sigma / R .
$$

The above quantity is non-zero for small $\zeta \in \mathbb{C} \backslash[0, \infty)$. By Lemma 4.1 and Lemma 4.2 (ii), $A_{\sigma}$ is bijective with inverse

$$
B_{\sigma}=B-\frac{\sigma}{\alpha_{\sigma}}\left\langle\cdot, B^{\times} p_{K}^{1}\right\rangle B 1=B+\frac{\sigma}{R(R+\sigma)}\left\langle\cdot, V W_{K}\right\rangle W_{K}
$$

after simplifying using Lemma 4.2 (iii) and (v). Now use

$$
\frac{\sigma}{R(R+\sigma)}=\frac{1}{R}-\frac{1}{R+\sigma}=\frac{1}{R}+\sum_{k=-\infty}^{-1} \theta_{k} \eta^{k}
$$

with $\theta_{k}$ as in (6.3). The expression (4.5) for $B$ leads to the result.

Now assume that $R=0$. In this case, $B_{\sigma}$ in (6.3) becomes

$$
B_{\sigma}=B_{0}-\frac{1}{\sigma}\left\langle\cdot, V W_{K}\right\rangle W_{K}
$$

For $u \in \mathscr{H}_{-s}$

$$
\begin{aligned}
B_{\sigma} A_{\sigma} u & =B_{0} A u+\sigma\left\langle u, p_{K}^{1}\right\rangle B_{0} 1-\frac{1}{\sigma}\left\langle A u, V W_{K}\right\rangle W_{K}-\left\langle u, p_{K}^{1}\right\rangle\left\langle 1, V W_{K}\right\rangle W_{K} \\
& =B_{0} A u+\left\langle u, p_{K}^{1}\right\rangle W_{K}
\end{aligned}
$$

by Lemma 4.5 (i) and Proposition 4.1 (v). Each $u \in \mathscr{H}_{-s}$ may be written uniquely in the form $u=v+\alpha W_{K}$ for some $v \in \mathscr{M}$ and $\alpha \in \mathbb{C}$. As $A W_{K}=0$ by Proposition 4.1 (iv), we obtain

$$
B_{\sigma} A_{\sigma} u=B_{0} A v+\alpha W_{K}=v+\alpha W_{K}=u
$$

by Lemma 4.6 (i). On the other hand,

$$
\begin{aligned}
A_{\sigma} B_{\sigma} u & =A B_{0} u+\sigma\left\langle B_{0} u, p_{K}^{1}\right\rangle 1-\frac{1}{\sigma}\left\langle u, V W_{K}\right\rangle A W_{K}-\left\langle u, V W_{K}\right\rangle\left\langle W_{K}, p_{K}^{1}\right\rangle 1 \\
& =A B_{0} u-\left\langle u, V W_{K}\right\rangle 1
\end{aligned}
$$

by Lemma 4.5 (ii) and Proposition 4.1 (iv). Each $u \in \mathscr{H}_{-s}$ may be written uniquely in the form $u=w+\beta 1$ for some $w \in \mathscr{W}$ and $\beta \in \mathbb{C}$. So

$$
A_{\sigma} B_{\sigma} u=A B_{0} w-\beta\left\langle 1, V W_{K}\right\rangle 1=w+\beta 1=u
$$

by Lemma 4.5 (i) and Lemma 4.6 (ii). This shows that $B_{\sigma}$ is the inverse of $A_{\sigma}$ in the case $R=0$. The final claim follows from the fact that $\sum_{k=-\infty}^{-1} \theta_{k} \eta^{k}=-\frac{1}{R+\sigma}$ is bounded for small $\zeta$.

Lemma 6.2. Let $l \in \mathbb{N}_{0}$ and $s>2 l+1$. Then for small $\lambda>0$,

$$
I+\sum_{j=1}^{l} \sum_{k=0}^{1} \lambda^{j} \eta^{k} B_{\sigma} A_{j}^{k} V=I-\sum_{j=1}^{l} \sum_{k=-\infty}^{1} \lambda^{j} \eta^{k} E_{j}^{k}
$$

in $B\left(\mathscr{H}_{-s}\right)$. The coefficients $E_{j}^{k}$ are given by

$$
\begin{array}{ll}
E_{j}^{1}=-B_{0} A_{j}^{1} V & \text { for } j=1,2, \ldots, \\
E_{j}^{0}=-B_{0} A_{j}^{0} V-\theta_{-1}\left\langle\cdot, V A_{j}^{1} V W_{K}\right\rangle W_{K} & \text { for } j=1,2, \ldots, \\
E_{j}^{k}=-\left\langle\cdot, V\left[\theta_{k} A_{j}^{0}+\theta_{k+1} A_{j}^{1}\right] V W_{K}\right\rangle W_{K} & \text { for } j=1,2, \ldots \text { and } k=-1,-2, \ldots
\end{array}
$$

The double-summation in 6.4) converges absolutely in norm. 
Proof. Replace the expression for $B_{\sigma}$ as in Proposition 6.1 to obtain

$$
\begin{aligned}
& I+ \sum_{j=1}^{l} \sum_{k=0}^{1} \lambda^{j} \eta^{k} B_{\sigma} A_{j}^{k} V=I+\sum_{j=1}^{l} \sum_{k=0}^{1} \lambda^{j} \eta^{k}\left\{B_{0}+\sum_{p=-\infty}^{-1} \theta_{p} \eta^{p}\left\langle\cdot, V W_{K}\right\rangle W_{K}\right\} A_{j}^{k} V \\
&= I+\sum_{j=1}^{l} \sum_{k=0}^{1} \lambda^{j} \eta^{k} B_{0} A_{j}^{k} V+\sum_{j=1}^{l} \sum_{k=0}^{1} \sum_{p=-\infty}^{-1} \lambda^{j} \eta^{k+p} \theta_{p}\left\langle\cdot, V A_{j}^{k} V W_{K}\right\rangle W_{K} \\
&=I+\sum_{j=1}^{l} \sum_{k=0}^{1} \lambda^{j} \eta^{k} B_{0} A_{j}^{k} V+\sum_{j=1}^{l} \sum_{p=-\infty}^{-1} \lambda^{j} \eta^{p} \theta_{p}\left\langle\cdot, V A_{j}^{0} V W_{K}\right\rangle W_{K}+\sum_{j=1}^{l} \sum_{p=-\infty}^{-1} \lambda^{j} \eta^{1+p} \theta_{p}\left\langle\cdot, V A_{j}^{1} V W_{K}\right\rangle W_{K} \\
&= \\
& \quad I+\sum_{j=1}^{l} \sum_{k=0}^{1} \lambda^{j} \eta^{k} B_{0} A_{j}^{k} V \\
& \quad+\sum_{j=1}^{l} \lambda^{j} \theta_{-1}\left\langle\cdot, V A_{j}^{1} V W_{K}\right\rangle W_{K} \\
&+\sum_{j=1}^{l} \sum_{k=-\infty}^{-1} \lambda^{j} \eta^{k}\left\{\theta_{k}\left\langle\cdot, V A_{j}^{0} V W_{K}\right\rangle W_{K}+\theta_{k-1}\left\langle\cdot, V A_{j}^{1} V W_{K}\right\rangle W_{K}\right\} \\
&= \\
& \quad+\sum_{j=1}^{l} \lambda^{j} \eta B_{0} A_{j}^{1} V+\sum_{j=1}^{l} \lambda^{j} B_{0} A_{j}^{0} V \\
&+\sum_{j=1}^{l} \lambda^{j} \theta_{-1}\left\langle\cdot, V A_{j}^{1} V W_{K}\right\rangle W_{K} \\
&+\sum_{j=1}^{l} \sum_{k=-\infty}^{-1} \lambda^{j} \eta^{k}\left\langle\cdot, V\left[\theta_{k} A_{j}^{0}+\theta_{k-1} A_{j}^{1}\right] V W_{K}\right\rangle W_{K} .
\end{aligned}
$$

From (6.3) it can be seen that there exist finite constants $e_{j}(j=1,2, \ldots)$ such that

$$
\left\|E_{j}^{k}\right\|_{B\left(\mathscr{H}_{-s}\right)} \leq e_{j} \theta^{-k}
$$

for $k=\ldots,-2,-1$. In fact, an estimate of the above form also extends to the case $k=0$ and $k=1$. This shows that the double-summation converges absolutely in norm.

Lemma 6.3. Let $l \in \mathbb{N}_{0}$ and $s>2 l+1$. Then

$$
\left\{I-\sum_{j=1}^{l} \sum_{k=-\infty}^{1} \lambda^{j} \eta^{k} E_{j}^{k}-o\left(\lambda^{l}\right)\right\}^{-1}=I+\sum_{j=1}^{l} \sum_{k=-\infty}^{j} \lambda^{j} \eta^{k} D_{j}^{k}+o\left(\lambda^{l}\right)
$$

in $B\left(\mathscr{H}_{-s}\right)$ as $\lambda \downarrow 0$. The coefficients $D_{j}^{k}$ are given by

$$
D_{j}^{k}=\sum_{|\alpha|=j,|\beta|=k} E_{\alpha}^{\beta}
$$

where the multi-indices $(\alpha, \beta)$ belong to the set

$$
(\alpha, \beta) \in \bigcup_{n=1}^{\infty} \mathbb{N}^{n} \times \Lambda^{n}
$$

where $\Lambda:=\{\ldots,-2,-1,0,1\}$. The double-summation in (6.6) converges absolutely in norm. 
Proof. The operator

$$
T:=\sum_{j=1}^{l} \sum_{k=-\infty}^{1} \lambda^{j} \eta^{k} E_{j}^{k}+o\left(\lambda^{l}\right)
$$

satisfies $\|T\|_{B\left(\mathscr{H}_{-s}\right)}<1$ for small $\lambda>0$. The inverse of $I-T$ may expressed as a Neumann series with coefficients as stated.

Suppose that $j \in \mathbb{N}$ and $\mathbb{Z} \ni k<0$. Using (6.5),

$$
\left\|D_{j}^{k}\right\|_{B\left(\mathscr{H}_{-s}\right)} \leq \sum_{n=1}^{j} \sum_{|\alpha|=j} \sum_{|\beta|=k}\left\|E_{\alpha}^{\beta}\right\|_{B\left(\mathscr{H}_{-s}\right)} \leq \theta^{-k} \sum_{n=1}^{j} \sum_{|\alpha|=j} e_{\alpha} a(n, k) .
$$

Assume that $k \leq-(3 / 2) j$. By Lemma 5.1, the right-hand side may be estimated via

$$
\left\{\sum_{n=1}^{j} \sum_{|\alpha|=j} 2^{n-1} a(n) e_{\alpha}\right\}|k|^{j-1} \theta^{-k}
$$

The index $n$ refers to the length of the multi-index $\alpha$. An inequality of the above form can be extended to the case $k<0$. In summary (for future use), for any $j \in \mathbb{N}$ and $k<0$,

$$
\left\|D_{j}^{k}\right\|_{B\left(\mathscr{H}_{-s}\right)} \leq d_{j}|k|^{j-1} \theta^{-k}
$$

for some finite constant $d_{j}$. This shows that the double-summation in (6.6) converges absolutely in norm.

Lemma 6.4. Let $l \in \mathbb{N}_{0}$ and $s>2 l+1$. Then

$$
\left(I+R^{(-1)}(\mu-\imath 0) V\right)^{-1}=\sum_{j=0}^{l} \sum_{k=-\infty}^{j} \lambda^{j} \eta^{k} B_{j}^{k}+o\left(\lambda^{l}\right)
$$

in $B\left(\mathscr{H}_{-s}\right)$ as $\lambda \downarrow 0$. The coefficients are given by

$$
\begin{array}{lll}
B_{0}^{0}=B_{0}, & \\
B_{0}^{k}=\theta_{k}\left\langle\cdot, V W_{K}\right\rangle W_{K} & & \text { for } k=-1,-2, \ldots, \\
B_{j}^{j}=D_{j}^{j} B_{0} & \text { for } j=1,2, \ldots, \\
B_{j}^{k}=D_{j}^{k} B_{0}+\sum_{p+q=k} \theta_{p}\left\langle\cdot, V W_{K}\right\rangle D_{j}^{q} W_{K} & & \text { for } j=1,2, \ldots \text { and } k<j .
\end{array}
$$

The double-summation in (6.8) converges absolutely in norm for small $\lambda>0$.

Proof. We rewrite (6.1) using Lemma 6.2 as

$$
I+R^{(-1)}(\mu-\imath 0) V=A_{\sigma}\left\{I-\sum_{j=1}^{l} \sum_{k=-\infty}^{1} \lambda^{j} \eta^{k} E_{j}^{k}-o\left(\lambda^{l}\right)\right\} .
$$


Inverting using Lemmas 6.3 and 6.1 we obtain

$$
\begin{aligned}
& \left(I+R^{(-1)}(\mu-\imath 0) V\right)^{-1}=B_{\sigma}+\sum_{j=1}^{l} \sum_{k=-\infty}^{j} \lambda^{j} \eta^{k} D_{j}^{k} B_{\sigma}+o\left(\lambda^{l}\right) \\
& =B_{0}+\sum_{k=-\infty}^{-1} \theta_{k} \eta^{k}\left\langle\cdot, V W_{K}\right\rangle W_{K} \\
& +\sum_{j=1}^{l} \sum_{k=-\infty}^{j} \lambda^{j} \eta^{k} D_{j}^{k} B_{0} \\
& +\sum_{j=1}^{l} \sum_{k=-\infty}^{j} \sum_{r=-\infty}^{-1} \lambda^{j} \eta^{k+r} \theta_{r}\left\langle\cdot, V W_{K}\right\rangle D_{j}^{k} W_{K}+o\left(\lambda^{l}\right) \\
& =B_{0}+\sum_{k=-\infty}^{-1} \theta_{k} \eta^{k}\left\langle\cdot, V W_{K}\right\rangle W_{K} \\
& +\sum_{j=1}^{l} \lambda^{j} \eta^{j} D_{j}^{j} B_{0} \\
& +\sum_{j=1}^{l} \sum_{k=-\infty}^{j-1} \lambda^{j} \eta^{k}\left\{D_{j}^{k} B_{0}+\sum_{p+q=k} \theta_{p}\left\langle\cdot, V W_{K}\right\rangle D_{j}^{q} W_{K}\right\}+o\left(\lambda^{l}\right) .
\end{aligned}
$$

For absolute convergence of the double-summation, let us first consider the term

$$
\sum_{p+q=k, q<0} \theta_{p}\left\langle\cdot, V W_{K}\right\rangle D_{j}^{q} W_{K}
$$

for $j \in \mathbb{N}$ and $k \leq-2$. From (6.7) its norm may be estimated by

$$
c \sum_{p+q=k, q<0}\left|\theta_{p}\right|\left\|D_{j}^{q}\right\|_{B\left(\mathscr{H}_{-s}\right)} \leq c^{\prime}\left\{\sum_{p+q=k, q<0}|q|^{j-1}\right\} \theta^{-k} \leq c^{\prime}|k|^{j} \theta^{-k}
$$

Consequently, for any $j \in \mathbb{N}_{0}$ and $k<0$,

$$
\left\|B_{j}^{k}\right\|_{B\left(\mathscr{H}_{-s}\right)} \leq b_{j}|k|^{j} \theta^{-k}
$$

for some finite constant $b_{j}$. So the double-summation converges absolutely in norm.

Proposition 6.2. Let $l \in \mathbb{N}_{0}$. There exist $T_{j}^{k} \in \mathfrak{S}_{1}(\mathfrak{h}), 0 \leq j \leq 2 l,-\infty<2 k \leq j$ such that

$$
T(\lambda)=\sum_{0 \leq j \leq 2 l} \sum_{-\infty<2 k \leq j}\left(\imath \lambda^{1 / 2}\right)^{j} \eta^{k} T_{j}^{k}+o\left(\lambda^{l}\right)
$$

in $\mathfrak{S}_{1}(\mathfrak{h})$ as $\lambda \downarrow 0$. The coefficients are given by

$$
T_{j}^{k}=2 \pi \imath \sum_{p+2 q+r=j}(-1)^{q+r} U_{p}^{(-1)} V B_{q}^{k} U_{r}^{(-1) *}
$$

for $0 \leq j \leq 2 l$ and $-\infty<2 k \leq j$. The double-summation in (6.11) converges absolutely in norm. Also,

$$
T_{0}^{0}=0 .
$$


Proof. The argument proceeds as in [18] Proposition 4.4. Choose $s>2 l+1$. As in (2.9),

$$
U^{(-1)}(\mu)=\sum_{p=0}^{2 l}\left(\imath \lambda^{1 / 2}\right)^{p} U_{p}^{(-1)}+o\left(\lambda^{l}\right)
$$

in $\mathfrak{S}_{2}\left(\mathscr{H}_{s}, \mathfrak{h}\right)$ as $\lambda \downarrow 0$. By Lemma 6.4

$$
\left(I+R^{(-1)}(\mu-\imath 0) V\right)^{-1}=\sum_{q=0}^{l} \sum_{k=-\infty}^{q}(-1)^{q}\left(\imath \lambda^{1 / 2}\right)^{2 q} \eta^{k} B_{q}^{k}+o\left(\lambda^{l}\right)
$$

in $B\left(\mathscr{H}_{-s}\right)$ as $\lambda \downarrow 0$. The expansion follows straightforwardly.

Fix $j \in \mathbb{N}_{0}$ and $k<0$. By (6.10),

$$
\left\|T_{j}^{k}\right\|_{\mathfrak{S}_{1}(\mathfrak{h})} \leq c \sum_{p+2 q+r=j}\left\|B_{q}^{k}\right\|_{B\left(\mathscr{H}_{-s}\right)} \leq c \sum_{p+2 q+r=j} b_{q}|k|^{q} \theta^{-k} \leq c^{\prime}\left\{\sum_{p+2 q+r=j} 1\right\}|k|^{[j / 2]} \theta^{-k}
$$

Thus for each $j \in \mathbb{N}_{0}$, there exists a finite constant $t_{j}$ such that

$$
\left\|T_{j}^{k}\right\|_{\mathfrak{S}_{1}(\mathfrak{h})} \leq t_{j}\langle k\rangle^{[j / 2]} \theta^{-k}
$$

for $-\infty<2 k \leq j$. This establishes the summability claim.

From (6.12), (2.11) and (2.7),

$$
T_{0}^{0}=2 \pi \imath U_{0} V B_{0} U_{0}^{*}=(\imath / 4 \pi)\left\langle V B_{0} 1,1\right\rangle\langle\cdot, 1\rangle .
$$

By Lemma 4.5 (i), $B_{0} 1=0$; hence $T_{0}^{0}=0$.

Theorem 6.1. Let $l \in \mathbb{N}$. Then

$$
\xi(\lambda)=\sum_{k=-l}^{-1} \xi_{0}^{k} \eta^{k}+O\left(\eta^{-(l+1)}\right)
$$

as $\lambda \downarrow 0$ where the coefficients are given by

$$
\xi_{0}^{k}=\frac{1}{2 \pi \imath} \sum_{|\alpha|=0,|\beta|=k} \frac{(-1)^{p}}{p} \operatorname{Tr}\left[T_{\alpha}^{\beta}\right] .
$$

In the above, $p$ signifies the length of the multi-index $\alpha$ (resp. $\beta$ ). Proof. For small $\lambda>0$,

$$
\xi(\lambda)=\frac{-1}{2 \pi \imath} \operatorname{Tr} \log (I+T(\lambda))
$$

in virtue of (2.17). From Proposition 6.2 we extract the expansion

$$
T(\lambda)=\sum_{k=-l}^{-1} \eta^{k} T_{0}^{k}+O\left(\eta^{-(l+1)}\right)
$$

and insert into the formula

$$
\log (I+T)=\sum_{p=1}^{\infty} \frac{(-1)^{p+1}}{p} T^{p}
$$

valid for $T \in B(\mathfrak{h})$ with $\|T\|<1$. 


\section{First three coefficients in low-energy expansion of the scattering phase}

Lemma 7.1. The following identities hold:

(i)

$$
\xi_{0}^{-1}=-\frac{1}{2 \pi \imath} \operatorname{Tr}\left[T_{0}^{-1}\right]
$$

(ii)

$$
\xi_{0}^{-2}=\frac{1}{2 \pi \imath}\left\{-\operatorname{Tr}\left[T_{0}^{-2}\right]+(1 / 2) \operatorname{Tr}\left[T_{0}^{-1} T_{0}^{-1}\right]\right\},
$$

(iii)

$$
\xi_{0}^{-3}=\frac{1}{2 \pi \imath}\left\{-\operatorname{Tr}\left[T_{0}^{-3}\right]+(1 / 2)\left(\operatorname{Tr}\left[T_{0}^{-2} T_{0}^{-1}\right]+\operatorname{Tr}\left[T_{0}^{-1} T_{0}^{-2}\right]\right)-(1 / 3) \operatorname{Tr}\left[T_{0}^{-1} T_{0}^{-1} T_{0}^{-1}\right]\right\} .
$$

Proof. These expressions follow directly from (6.14).

Theorem 7.1. The following identities hold:

(i) $\xi_{0}^{-1}=1$;

(ii) $\xi_{0}^{-2}=C(K)-\log 4+2 \gamma$;

(iii) $\xi_{0}^{-3}=(C(K)-\log 4+2 \gamma)^{2}-\frac{\pi^{2}}{3}$.

Proof. First note that from (6.9) the identity $\left\langle 1, V B_{0}^{k} 1\right\rangle=\theta_{k}$ holds for any $\mathbb{Z} \ni k<0$. Moreover, from (6.12),

$$
T_{0}^{k}=2 \pi \imath U_{0} V B_{0}^{k} U_{0}^{*}
$$

for any $k<0$. With the help of [19] Corollary 7.2 (i) (or straightforwardly from (2.7)),

$$
\begin{aligned}
\operatorname{Tr}\left[T_{0}^{k}\right] & =2 \pi \imath \operatorname{Tr}\left[U_{0} V B_{0}^{k} U_{0}^{*}\right] \\
& =2 \pi \imath(1 / 4 \pi)\left\langle V B_{0}^{k} 1,1\right\rangle \\
& =(\imath / 2) \theta_{k} .
\end{aligned}
$$

From (6.3) we have

$$
\begin{aligned}
& \theta_{-1}=-\frac{1}{b}, \\
& \theta_{-2}=\frac{1}{b}\left(\frac{R+a}{b}\right), \\
& \theta_{-3}=-\frac{1}{b}\left(\frac{R+a}{b}\right)^{2}
\end{aligned}
$$

with

$$
a=(1 / 2 \pi)(\log 2-\gamma)+\imath / 4 \text { and } b=1 / 4 \pi \text {. }
$$

(i) From (7.1),

$$
\operatorname{Tr}\left[T_{0}^{-1}\right]=(\iota / 2) \theta_{-1}=-2 \pi \imath .
$$

This and Lemma 7.1 (i) gives the first item.

(ii) Using the above identity once more,

$$
\operatorname{Tr}\left[T_{0}^{-2}\right]=(\imath / 2) \theta_{-2} .
$$


With the help of [19] Corollary 7.2 (vii),

$$
\begin{aligned}
\operatorname{Tr}\left[T_{0}^{-1} T_{0}^{-1}\right] & =(2 \pi \imath)^{2} \operatorname{Tr}\left[U_{0} V B_{0}^{-1} U_{0}^{*} U_{0} V B_{0}^{-1} U_{0}^{*}\right] \\
& =-(2 \pi)^{2}(1 / 4)(2 \pi)^{-4}(2 \pi)^{2}\left\langle V B_{0}^{-1} 1,1\right\rangle^{2} \\
& =-(1 / 4) \theta_{-1}^{2} .
\end{aligned}
$$

By Lemma 7.1 (ii),

$$
\xi_{0}^{-2}=\frac{1}{2 \pi \imath}\left\{-(1 / 2) \imath \theta_{-2}-(1 / 8) \theta_{-1}^{2}\right\}=-4 \pi[R+\Re a]=C(K)-\log 4+2 \gamma .
$$

(iii) From (7.1),

$$
\operatorname{Tr}\left[T_{0}^{-3}\right]=(\imath / 2) \theta_{-3} .
$$

By [19] Corollary 7.2 (vii),

$$
\begin{aligned}
\operatorname{Tr}\left[T_{0}^{-2} T_{0}^{-1}\right] & =(2 \pi \imath)^{2} \operatorname{Tr}\left[U_{0} V B_{0}^{-2} U_{0}^{*} U_{0} V B_{0}^{-1} U_{0}^{*}\right] \\
& =-(2 \pi)^{2}(1 / 4)(2 \pi)^{-4}(2 \pi)^{2}\left\langle V B_{0}^{-2} 1,1\right\rangle\left\langle V B_{0}^{-1} 1,1\right\rangle \\
& =-(1 / 4) \theta_{-2} \theta_{-1} .
\end{aligned}
$$

By [19] Corollary 7.2 (viii),

$$
\begin{aligned}
\operatorname{Tr}\left[T_{0}^{-1} T_{0}^{-1} T_{0}^{-1}\right] & =(2 \pi \imath)^{3} \operatorname{Tr}\left[U_{0} V B_{0}^{-1} U_{0}^{*} U_{0} V B_{0}^{-1} U_{0}^{*} U_{0} V B_{0}^{-1} U_{0}^{*}\right] \\
& =-\imath(2 \pi)^{3}(1 / 8)(2 \pi)^{-6}(2 \pi)^{3}\left\langle V B_{0}^{-1} 1,1\right\rangle^{3} \\
& =-(\imath / 8) \theta_{-1}^{3} .
\end{aligned}
$$

By Lemma 7.1 (iii) and some computation,

$$
\begin{aligned}
\xi_{0}^{-3} & =\frac{1}{2 \pi}\left\{-(\imath / 2) \theta_{-3}-(1 / 4) \theta_{-2} \theta_{-1}-(1 / 3)(-\imath / 8) \theta_{-1}^{3}\right\} \\
& =(4 \pi)^{2}\left\{(R+a-\imath / 4)^{2}-\frac{1}{48}\right\} \\
& =(C(K)-\log 4+2 \gamma)^{2}-\frac{\pi^{2}}{3} .
\end{aligned}
$$

\section{Asymptotics of the pinned Wiener sausage}

We first remark that $\gamma(t)$ may be written purely analytically as

$$
\gamma(t)=(4 \pi t) \operatorname{Tr}\left[e^{-t H}-e^{-t H_{Y}}\right] .
$$

Let $0<\delta<1$. For $k \in \mathbb{Z}$,

$$
\int_{0}^{\delta} t e^{-t \lambda}(-\log \lambda)^{k} d \lambda \sim \sum_{r=0}^{\infty}(-1)^{r}\left(\begin{array}{l}
k \\
r
\end{array}\right) \Gamma^{(r)}(1)(\log t)^{k-r}
$$

as $t \rightarrow \infty$ according to [9] Lemma 3 . Recall that for $k<0$, the binomial is specified by

$$
\left(\begin{array}{l}
k \\
r
\end{array}\right)=(-1)^{r}\left(\begin{array}{c}
-k+r-1 \\
r
\end{array}\right)
$$

Theorem 8.1. Let $l \in \mathbb{N}$. Then

$$
\gamma(t)=\sum_{k=-l}^{-1} \gamma_{0}^{k} t(\log t)^{k}+o\left(t(\log t)^{-l}\right)
$$

as $t \rightarrow \infty$ where

$$
\gamma_{0}^{k}=4 \pi \sum_{s-r=k} \xi_{0}^{s}(-1)^{r}\left(\begin{array}{l}
s \\
r
\end{array}\right) \Gamma^{(r)}(1) .
$$

The extra constraints $-\infty<s \leq-1$ and $r \geq 0$ apply in the summation. 
Proof. We write

$$
\gamma(t)=(4 \pi t)\left\{\int_{0}^{\delta} t e^{-t \lambda} \xi(\lambda) d \lambda+\int_{\delta}^{\infty} t e^{-t \lambda} \xi(\lambda) d \lambda\right\} .
$$

In virtue of (2.13) the second term decays exponentially. Write

$$
\xi(\lambda)=\sum_{k=-l}^{-1} \xi_{0}^{k} \eta^{k}+O\left(\eta^{-(l+1)}\right)
$$

according to Theorem 6.1, By (8.2) the term

$$
(4 \pi t) \int_{0}^{\delta} t e^{-t \lambda} \eta^{-(l+1)} d \lambda=o\left(t(\log t)^{-l}\right)
$$

can be absorbed into the remainder. Again by (8.2), for $-l \leq k \leq-1$,

$$
(4 \pi t) \int_{0}^{\delta} t e^{-t \lambda} \eta^{k} d \lambda=4 \pi \sum_{r=0}^{k+l}(-1)^{r}\left(\begin{array}{l}
k \\
r
\end{array}\right) \Gamma^{(r)}(1) t(\log t)^{k-r}+o\left(t(\log t)^{-l}\right)
$$

as $t \rightarrow \infty$. Therefore,

$$
\begin{aligned}
(4 \pi t) \int_{0}^{\delta} t e^{-t \lambda} \sum_{k=-l}^{-1} \xi_{0}^{k} \eta^{k} d \lambda & =\sum_{k=-l}^{-1} \xi_{0}^{k}(4 \pi t) \int_{0}^{\delta} t e^{-t \lambda} \eta^{k} d \lambda \\
& =\sum_{k=-l}^{-1} \xi_{0}^{k} 4 \pi \sum_{r=0}^{k+l}(-1)^{r}\left(\begin{array}{l}
k \\
r
\end{array}\right) \Gamma^{(r)}(1) t(\log t)^{k-r}+o\left(t(\log t)^{-l}\right) \\
& =\sum_{k=-l}^{-1}\left\{4 \pi \sum_{s-r=k}(-1)^{r} \xi_{0}^{s}\left(\begin{array}{l}
s \\
r
\end{array}\right) \Gamma^{(r)}(1)\right\} t(\log t)^{k}+o\left(t(\log t)^{-l}\right) .
\end{aligned}
$$

It is understood that $-\infty<s \leq-1$ and $r \geq 0$ in the summation.

Corollary 8.1. The following identities hold:
(i) $\gamma_{0}^{-1}=4 \pi$;
(ii) $\gamma_{0}^{-2}=4 \pi\{C(K)+\gamma-\log 4\}$;
(iii) $\gamma_{0}^{-3}=4 \pi\left\{(C(K)+\gamma-\log 4)^{2}-\frac{\pi^{2}}{6}\right\}$.

Proof. From (8.3) we derive

$$
\begin{aligned}
& \text { (a) } \gamma_{0}^{-1}=4 \pi \xi_{0}^{-1} \\
& \text { (b) } \gamma_{0}^{-2}=4 \pi\left\{\xi_{0}^{-1} \Gamma^{(1)}(1)+\xi_{0}^{-2}\right\} ; \\
& \text { (c) } \gamma_{0}^{-3}=4 \pi\left\{\xi_{0}^{-1} \Gamma^{(2)}(1)+2 \xi_{0}^{-2} \Gamma^{(1)}(1)+\xi_{0}^{-3}\right\} .
\end{aligned}
$$

According to [1] 6.4.2,

$$
\Gamma^{(1)}(1)=-\gamma, \quad \Gamma^{(2)}(1)=\gamma^{2}+\frac{\pi^{2}}{6} .
$$

The identities (i)-(iii) now follow with the help of Theorem 7.1

Acknowledgement. I would like to express my thanks to Professor Michiel van den Berg for several helpful conversations on the topic of this paper and also for suggesting this problem in the first place. For this, I am also grateful to the anonymous referee of [19]. 


\section{Appendix}

In this Appendix we prove Theorem 2.1, Lemma 2.1 and Theorem 2.2.

Lemma 9.1. The operator $K$ with convolution kernel

$$
k(x):=(\log |x|)|x|^{\alpha} \quad(-2<\alpha<\infty)
$$

belongs to $\mathfrak{S}_{2}\left(\mathscr{H}_{s}, \mathscr{H}_{-s}\right)$ whenever $s>\alpha \vee 0+1$.

Proof. Consider the operator $K$ with convolution kernel $k(x):=|x|^{\alpha}$. Suppose that $\alpha \geq 0$. For $s>\alpha+1$

$$
\begin{aligned}
\|K\|_{\mathfrak{S}_{2}\left(\mathscr{H}_{s}, \mathscr{H}_{-s}\right)}^{2} & =\int_{\mathbb{R}^{2} \times \mathbb{R}^{2}}\langle x\rangle^{-2 s}|x-y|^{2 \alpha}\langle y\rangle^{-2 s} d y d x \\
& \leq 4^{\alpha} \int_{\mathbb{R}^{2} \times \mathbb{R}^{2}}\langle x\rangle^{-2 s+2 \alpha}\langle y\rangle^{-2 s+2 \alpha} d y d x<\infty .
\end{aligned}
$$

In case the kernel $k$ includes the logarithmic term, split the integral into a sum of integrals over the domains

$$
A_{1}:=\left\{(x, y) \in \mathbb{R}^{2} \times \mathbb{R}^{2}: 0<|x-y|<1\right\} \text { and } A_{2}:=\left\{(x, y) \in \mathbb{R}^{2} \times \mathbb{R}^{2}:|x-y|>1\right\} .
$$

On $A_{1}$ use Young's inequality ([ 8$]$ 1.1.4) and on $A_{2}$ use the inequality

$$
\log |x-y| \leq 2^{\varepsilon} \varepsilon^{-1}\langle x\rangle^{\varepsilon}\langle y\rangle^{\varepsilon}
$$

valid for any $\varepsilon>0$. The above decomposition can also be used to deal with the case $-2<\alpha<$ 0 .

Lemma 9.2. Let $\beta>2$. For $y \in \mathbb{R}^{2}$ and $0<r \leq 1 / 2$ define

$$
f(y, r):=\int_{|x-y| \geq r^{-1}}\langle x\rangle^{-\beta} d x
$$

Then there exists a finite constant $c$ such that

$$
f(y, r) \leq\left\{\begin{array}{lll}
c r^{\beta-2} & \text { for } & |y| \leq \frac{1}{2 r} \\
c & \text { for } & |y|>\frac{1}{2 r}
\end{array}\right.
$$

Proof. The result for $|y|>1 / 2 r$ is clear. Suppose that $|y| \leq 1 / 2 r$. Then $1-r|y| \geq 1 / 2 \geq r$. Thus, $B\left(0, r^{-1}-|y|\right) \subseteq B\left(y, r^{-1}\right)$ and $B(0,1 / 2) \subseteq B(0,1-r|y|)$. This means that

$$
f(y, r) \leq \int_{|x| \geq r^{-1}-|y|}\langle x\rangle^{-\beta} d x \leq r^{\beta-2} \int_{|x| \geq 1 / 2}|x|^{-\beta} d x .
$$

Let $\varphi_{1}$ denote the indicator function of the interval $[0,1]$ and $\varphi_{2}:=1-\varphi_{1}$.

Lemma 9.3. Let $K(\zeta)$ be the operator with convolution kernel

$$
k(x ; \zeta):=\varphi_{2}\left(|\zeta|^{1 / 2}|x|\right)(\log |x|)|x|^{\alpha} \quad(\zeta \in \mathbb{C} \backslash[0, \infty), \alpha \in \mathbb{R}) .
$$

Let $s>\alpha \vee 0+1$. Then $K(\zeta)$ belongs to $\mathfrak{S}_{2}\left(\mathscr{H}_{s}, \mathscr{H}_{-s}\right)$ and

$$
\|K(\zeta)\|_{\mathfrak{S}_{2}\left(\mathscr{H}_{s}, \mathscr{H}_{-s}\right)}=O\left(|\zeta|^{(s-\alpha-1) / 2}\right)
$$

as $\zeta \rightarrow 0$. 
Proof. Consider the operator $K(\zeta)$ with convolution kernel $k(x ; \zeta):=\varphi_{2}\left(|\zeta|^{1 / 2}|x|\right)|x|^{\alpha}$. Suppose that $\alpha \geq 0$. For $s>\alpha+1$ we find

$$
\|K(\zeta)\|_{\mathfrak{S}_{2}\left(\mathscr{H}_{s}, \mathscr{H}_{-s}\right)}^{2} \leq 4^{\alpha} \int_{\mathbb{R}^{2}}\langle y\rangle^{-\beta} f\left(y,|\zeta|^{1 / 2}\right) d y
$$

where $f$ is defined as in Lemma 9.2 and $\beta:=2(s-\alpha)$ and $r:=|\zeta|^{1 / 2}$. Using the estimate in Lemma 9.2 this may be bounded by

$$
4^{\alpha} c\left\{|\zeta|^{s-\alpha-1} \int_{|y| \leq 1 / 2|\zeta|^{1 / 2}}\langle y\rangle^{-\beta} d y+\int_{|y|>1 / 2|\zeta|^{1 / 2}}\langle y\rangle^{-\beta} d y\right\}
$$

for $0<|\zeta| \leq 1 / 4$. The latter integral has order $O\left(|\zeta|^{s-\alpha-1}\right)$ as $\zeta \rightarrow 0$. This gives the result for $\alpha \geq 0$. Now suppose that $\alpha<0$. For $s>1$,

$$
\|K(\zeta)\|_{\mathfrak{S}_{2}\left(\mathscr{H}_{s}, \mathscr{H}_{-s}\right)}^{2} \leq|\zeta|^{-\alpha} \int_{|x-y| \geq|\zeta|^{-1 / 2}}\langle x\rangle^{-2 s}\langle y\rangle^{-2 s} d y d x .
$$

Combining this with the result for $\alpha=0$ yields the result for this case. In case the kernel $k(\cdot ; \zeta)$ includes the logarithmic term, make use of (9.1).

Lemma 9.4. Let $K(\zeta)$ be the operator with convolution kernel

$$
k(x ; \zeta):=\varphi_{1}\left(|\zeta|^{1 / 2}|x|\right)|x|^{\alpha} \quad(\zeta \in \mathbb{C} \backslash[0, \infty), \alpha>0) .
$$

Let $\alpha<s \leq \alpha+1$ with $s>1$. Then $K(\zeta)$ belongs to $\mathfrak{S}_{2}\left(\mathscr{H}_{s}, \mathscr{H}_{-s}\right)$ and

$$
\|K(\zeta)\|_{\mathfrak{S}_{2}\left(\mathscr{H}_{s}, \mathscr{H}_{-s}\right)}= \begin{cases}O\left(|\zeta|^{(s-\alpha-1) / 2}\right) & \text { if } \quad \alpha<s<\alpha+1, \\ O\left((-\log |\zeta|)^{1 / 2}\right) & \text { if } \quad s=\alpha+1,\end{cases}
$$

as $\zeta \rightarrow 0$.

Proof. Using the fact that

$$
|x-y|^{2 \alpha} \leq 2^{2 \alpha}\langle y\rangle^{2 \alpha} \text { for }|y| \geq|x|
$$

we have

$$
\begin{aligned}
\|K(\zeta)\|_{\mathfrak{S}_{2}\left(\mathscr{H}_{s}, \mathscr{H}_{-s}\right)}^{2} & =\int_{|x-y| \leq|\zeta|^{-1 / 2}}\langle x\rangle^{-2 s}|x-y|^{2 \alpha}\langle y\rangle^{-2 s} d y d x \\
& \leq 2^{2 \alpha+1} \int_{\mathbb{R}^{2}}\langle x\rangle^{-2 s} \int_{|x-y| \leq|\zeta|^{-1 / 2}}\langle y\rangle^{2(\alpha-s)} d y d x \\
& \leq 2^{2 \alpha+1} \int_{\mathbb{R}^{2}}\langle x\rangle^{-2 s} \int_{|y| \leq|\zeta|^{-1 / 2}}\langle y\rangle^{2(\alpha-s)} d y d x .
\end{aligned}
$$

For $\alpha<s<\alpha+1$,

$$
\int_{|y| \leq|\zeta|^{-1 / 2}}\langle y\rangle^{2(\alpha-s)} d y \leq \frac{\pi}{\alpha-s+1} 4^{\alpha-s+1}|\zeta|^{s-\alpha-1}
$$

for $0<|\zeta|<1$. On the other hand, for $s=\alpha+1$,

$$
\int_{|y| \leq|\zeta|^{-1 / 2}}\langle y\rangle^{2(\alpha-s)} d y \leq 2 \pi\left\{-\frac{1}{2} \log |\zeta|+\log 2\right\}
$$

again for $0<|\zeta|<1$. This leads to the result. 
Proof of Theorem [2.1. First recall that by [1] 9.2.3,

$$
\left|H_{0}^{(1)}(z)\right| \leq c|z|^{-1 / 2} \text { for }|z|>1 \text { and } 0<\operatorname{Arg} z<\pi .
$$

With $\varphi_{1}, \varphi_{2}$ as before set

$$
k^{(j)}(x ; \zeta):=\varphi_{j}\left(|\zeta|^{1 / 2}|x|\right) k(x ; \zeta) \quad(j=1,2)
$$

with $k(\cdot ; \zeta)$ as in (2.6). Using Lemma 9.1 and the estimate (9.2) for $k^{(2)}(x ; \zeta)$ it may be seen that $R(\zeta)$ belongs to $\mathfrak{S}_{2}\left(\mathscr{H}_{s}, \mathscr{H}_{-s}\right)$ for any $s>1$.

Let $l \in \mathbb{N}_{0}$ and $s>2 l+1$. By Lemma 9.1 each of the operators $K_{j}^{\varepsilon}$ belongs to $\mathfrak{S}_{2}\left(\mathscr{H}_{s}, \mathscr{H}_{-s}\right)$ for $j=0, \ldots, l$ and $\varepsilon=0,1$. Define

$$
k_{l}(x ; \zeta):=\sum_{j=0}^{l} \sum_{\varepsilon=0}^{1} \zeta^{j} \eta^{\varepsilon} k_{j}^{\varepsilon}(x) \quad\left(x \in \mathbb{R}^{2} \backslash\{0\}\right)
$$

and the cut-off kernels $k_{l}^{(j)}(\cdot ; \zeta)(j=1,2)$ as above. By (2.2) there exists a finite constant $c$ such that

$$
\left|k^{(1)}(x ; \zeta)-k_{l}^{(1)}(x ; \zeta)\right| \leq c \varphi_{1}\left(|\zeta|^{1 / 2}|x|\right)|\zeta|^{l+1}|\eta|(1+|\log | x||)|x|^{l+1}
$$

for small $\zeta$. Set $\alpha=l+1$. For $l \geq 1$ we have that $s>\alpha+1$. The remainder estimate follows from Lemma 9.1. Consider the case $l=0$. If $s>2$ use Lemma 9.1. If $1<s \leq 2$ use Lemma 9.4 To deal with the logarithmic term consider the operators with kernels

$$
\varphi_{j}(|x|)\left\{k^{(1)}(x ; \zeta)-k_{l}^{(1)}(x ; \zeta)\right\} \quad(j=1,2) .
$$

The operator corresponding to $j=1$ is bounded by Young's inequality. For the second use the fact that for any $\varepsilon>0$ there exists a finite constant $c$ such that

$$
\varphi_{2}(|x|) \log |x| \leq c|x|^{\varepsilon} \text { for } x \in \mathbb{R}^{2} .
$$

In view of (9.2) we have that

$$
\left|k^{(2)}(x ; \zeta)\right| \leq c|\zeta|^{-1 / 4} \varphi_{2}\left(|\zeta|^{1 / 2}|x|\right)|x|^{-1 / 2}
$$

and by Lemma 9.3 we obtain that

$$
\left\|K^{(2)}(\zeta)\right\|_{\mathfrak{S}_{2}\left(\mathscr{H}_{s}, \mathscr{H}_{-s}\right)}=O\left(|\zeta|^{\frac{s-1}{2}}\right)=o\left(|\zeta|^{l}\right) .
$$

Similar considerations can be used to deal with the terms in $K_{l}^{(2)}(\zeta)$.

Proof of Lemma 2.1. The kernel of $U(\lambda)$ is given by

$$
u(\omega, x ; \lambda)=\frac{1}{\sqrt{2}}(2 \pi)^{-1} e^{-\imath \lambda^{1 / 2} \omega \cdot x} .
$$

Therefore, $u(\omega, x ; \lambda)$ has an absolutely convergent series expansion of the form

$$
u(\omega, x ; \lambda)=\sum_{j=0}^{\infty}\left(\imath \lambda^{1 / 2}\right)^{j} u_{j}(\omega, x)
$$

where

$$
u_{j}(\omega, x):=\frac{1}{\sqrt{2}}(2 \pi)^{-1} \frac{(-1)^{j}}{j !}(\omega \cdot x)^{j} .
$$


It is clear that $u(\omega, x ; \lambda)$ is uniformly bounded. The truncated kernel will be written $u_{l}(\omega, x ; \lambda)$. Let $\varphi_{1}, \varphi_{2}$ be as previously. Define

$$
u^{(j)}(\omega, x ; \lambda):=\varphi_{j}\left(\lambda^{1 / 2}|x|\right) u(\omega, x ; \lambda) \quad(j=1,2)
$$

and $u_{l}^{(j)}(\omega, x ; \lambda)$ similarly. Denote the corresponding operators by $U^{(1)}(\lambda)$, etc. Let $U$ be the operator with kernel $u(\omega, x):=|x|^{j}\left(j \in \mathbb{N}_{0}\right)$. Note that $U \in \mathfrak{S}_{2}\left(\mathscr{H}_{s}, \mathfrak{h}\right)$ if $s>j+1$. Let $U^{(j)}(\lambda)$ be the operator with kernel

$$
u^{(j)}(\omega, x ; \lambda):=\varphi_{j}\left(\lambda^{1 / 2}|x|\right)|x|^{2 j} .
$$

Then $U^{(1)}(\lambda)$ has Hilbert-Schmidt norm $O\left(\lambda^{(s-j-1) / 2}\right)$ provided $s \leq j+1$. On the other hand, the operator $U^{(2)}(\lambda)$ has norm $O\left(\lambda^{(s-j-1) / 2}\right)$ if $s>j+1$.

We have the estimate

$$
\left|u^{(1)}(\omega, x ; \lambda)-u_{l}^{(1)}(\omega, x ; \lambda)\right| \leq c\left(\lambda^{1 / 2}|x|\right)^{l+1} .
$$

Thus $\left\|U^{(1)}(\lambda)-U_{l}^{(1)}(\lambda)\right\|_{\mathfrak{S}_{2}\left(\mathscr{H}_{s}, \mathfrak{h}\right)}=o\left(\lambda^{l / 2}\right)$ provided $s>l+1$. It is straightforward to see that the Hilbert-Schmidt norm of the difference $U^{(2)}(\lambda)-U_{l}^{(2)}(\lambda)$ admits an estimate of the same order in $\lambda$.

Proof of Theorem 2.2. The compactness statement is equivalent to the result $\langle\cdot\rangle^{s} V\langle\cdot\rangle^{s} \in \mathfrak{S}_{\infty}(\mathscr{H})$. Define $V(t):=J e^{-t H_{Y}} J^{*}-e^{-t H}$. Then

$$
\langle\cdot\rangle^{s} V(2 t)\langle\cdot\rangle^{s}=\langle\cdot\rangle^{s} V(t) e^{-t H}\langle\cdot\rangle^{s}+\langle\cdot\rangle^{s} J e^{-t H_{Y}} J^{*} V(t)\langle\cdot\rangle^{s} .
$$

The kernel $k(t ; x, y)$ of $\langle\cdot\rangle^{-s} e^{-t H}\langle\cdot\rangle^{s}$ is well-known to be

$$
k(t ; x, y)=(4 \pi t)^{-1}\langle x\rangle^{-s} e^{-|x-y|^{2} / 4 t}\langle y\rangle^{s} .
$$

Using the inequality

$$
\langle y\rangle^{s} \leq 2^{s}\left(\langle x\rangle^{s}+\langle x-y\rangle^{s}\right)
$$

we see that $k(t ; x, y)$ is dominated by a square-integrable convolution kernel and hence by Young's inequality ([8] 1.1.4 for example) $\langle\cdot\rangle^{-s} e^{-t H}\langle\cdot\rangle^{s} \in B\left(\mathscr{H}, L^{\infty}\left(\mathbb{R}^{2}\right)\right)$. Let $M=\left(\Omega, \mathscr{M}, X_{t}, \mathbb{P}_{x}\right)$ be Brownian motion on $\mathbb{R}^{2}$. Put $\sigma_{K}:=\inf \left\{t>0: X_{t} \in K\right\}$, the first hitting time of $K$. By the strong Markov property of Brownian motion,

$$
\begin{aligned}
\left|\langle\cdot\rangle^{s} V(t)\langle\cdot\rangle^{s} 1(x)\right| & =\langle x\rangle^{s} \mathbb{E}_{x}\left(\left\langle X_{t}\right\rangle^{s}: \sigma(K)<t\right) \\
& =\langle x\rangle^{s} \mathbb{E}_{x}\left(\mathbb{E}_{X_{\sigma(K)}}\left\langle X_{t-\sigma(K)}\right\rangle^{s}: \sigma(K)<t\right) .
\end{aligned}
$$

Since

$$
\sup _{y \in K} \sup _{0 \leq \tau \leq t} \mathbb{E}_{y}\left\langle X_{\tau}\right\rangle^{s}
$$

is finite, (9.3) is square-integrable. Therefore, $\langle\cdot\rangle^{s} V(t)\langle\cdot\rangle^{s} \in B\left(L^{\infty}\left(\mathbb{R}^{2}\right), \mathscr{H}\right)$. We conclude that $\langle\cdot\rangle^{s} V(t) e^{-t H}\langle\cdot\rangle^{s} \in \mathfrak{S}_{2}(\mathscr{H})$ (see [24] for example) and hence the same for $\langle\cdot\rangle^{s} V(2 t)\langle\cdot\rangle^{s}$ by domination and duality.

Compactness of $\langle\cdot\rangle^{s} V\langle\cdot\rangle^{s}$ follows once we have shown that

$$
\int_{0}^{\infty} e^{-t}\left\|\langle\cdot\rangle^{s} V\langle\cdot\rangle^{s}\right\|_{B(\mathscr{H})} d t<\infty
$$

by [29] Theorem 1.3 and Remark 1.2 (b). Applying Hölder's inequality inside the functional integral we obtain for any $f \in \mathscr{H}$,

$$
\left\|\langle\cdot\rangle^{s} V(t)\langle\cdot\rangle^{s} f\right\|_{2} \leq \sup _{y \in K} \sup _{0 \leq \tau \leq t}\left(\mathbb{E}_{y}\left\langle X_{\tau}\right\rangle^{2 s}\right)^{1 / 2} \sup _{x \in \mathbb{R}^{2}}\langle x\rangle^{s} \mathbb{P}_{x}(\sigma(K)<t)^{1 / 2}\|f\|_{2} .
$$


The known expression for the Brownian motion transition density yields that

$$
t \mapsto \sup _{y \in K} \sup _{0 \leq \tau \leq t}\left(\mathbb{E}_{y}\left\langle X_{\tau}\right\rangle^{2 s}\right)^{1 / 2}
$$

is $O(1)$ as $t \rightarrow 0+$ and $O\left(t^{s / 2}\right)$ as $t \rightarrow \infty$. The function

$$
t \mapsto \sup _{x \in \mathbb{R}^{2}}\langle x\rangle^{s} \mathbb{P}_{x}(\sigma(K)<t)^{1 / 2}
$$

has the same behaviour as can be seen using the "principle of not feeling the boundary". Thus the above integral is indeed finite.

\section{References}

[1] Abramowitz, M. and Stegun, A., Handbook of mathematical functions, National Bureau of Standards Applied Mathematics Series 55, 1964.

[2] D.R. Adams, and L.I. Hedberg, Function spaces and potential theory, Springer, 1996.

[3] Agmon, S., Spectral properties of Schrödinger operators and scattering theory, Ann. Scuola Norm. Pisa Cl. Sci. (4) II, No. 2, (1995)

[4] Bollé, D., Gesztesy, F., Danneels, C., Threshold scattering in two dimensions, Annales Inst. H. Poincaré Phys. Théor. 48 (1988), no. 2, 175 - 204.

[5] van den Berg, M. and Bolthausen, E., On the expected volume of the Wiener sausage for a Brownian bridge, Math. Zeit. 224 (1997), 33-48.

[6] Davies, E.B., Heat kernels and spectral theory, Cambridge University Press. 1989.

[7] Demuth, M. and McGillivray, I., Capacitary estimates for the scattering phase, J. Math. Anal. Appl. 237 (1999), no. 1, 253-271.

[8] Davies, E.B., Heat kernels and spectral theory, Cambridge University Press, 1989.

[9] Erdélyi, A., General asymptotic expansions of Laplace integrals, Arch. Rat. Mech. Anal. 7, No. 1 (1960), 1-20

[10] Fukushima, M., Dirichlet forms and Markov processes, Kodansha, 1980.

[11] Hörmander, L., Uniqueness theorems for second order elliptic differential equations, Comm. Partial Differential Equations 8 (1) (1983), 21 - 64.

[12] Le Gall, J.F., Sur une conjecture de M. Kac, Probab. Th. Rel. Fields 78 (1988), 389 - 402.

[13] Le Gall, J.F., Wiener sausage and self-intersection local times, J. Funct. Anal. 88 (1990). 299-341.

[14] Gesztesy, F., On stationary two-body scattering theory in two dimensions in Models and methods in few-body physics. Springer, 1987.

[15] Hassell, A. and Zelditch, S. Determinants of Laplacians in exterior domains, Internat. Math. Res. Notices. 18 (1999), 971-1004.

[16] Jensen, A. and Kato, T. Asymptotic behaviour of the scattering phase in exterior domains, Comm. Partial Differential Equations 3 (12) (1978), 1165 - 1195.

[17] Lieb, E. and Loss, M., Analysis. American Mathematical Society. 1997. 
[18] McGillivray, I., Large time volume of the pinned Wiener sausage, J. Funct. Anal. 170 (2000). $107-140$.

[19] McGillivray, I., Large time volume of the pinned Wiener sausage to second order, Math. Nachr. 2011, 284, No. 02-3, 142-165.

[20] Port, S. and Stone, C., Brownian motion and classical potential theory, Academic Press. 1978.

[21] Port, S., Spitzer's formula involving capacity, in Progress in Probability, Vol. 28. Birkhäuser, 1991.

[22] Reed, M. and Simon, B., Methods of modern mathematical physics II: Fourier analysis, selfadjointness. Academic Press. 1975

[23] Spitzer, F., Electrostatic capacity, heat flow and Brownian motion, Z. für Wahrscheinlichkeitstheorie Verw. Geb. 3 (1964). 187-197.

[24] Stollmann, P., Scattering at obstacles of finite capacity, J. Funct. Anal. 121 (1994), 416-425

[25] Taylor, M., Partial Differential Equations. Basic Theory. Springer-Verlag. 1996.

[26] Taylor, M., Partial Differential Equations. Qualitative Studies of Linear Equations. SpringerVerlag. 1996.

[27] Thorpe, J.A., Elementary topics in differential geometry, Springer. 1976.

[28] Uhlenbeck, G.E. and Beth, E., The quantum theory of the non-ideal gas I. Deviations from the classical theory, Physica III 8 (1936), 729 - 745.

[29] Voigt, J., On the convex compactness property for the strong operator topology, Note Mat. 12 (1992), 259-269

[30] Widder, D.V., The Laplace transform, Princeton University Press. 1946.

[31] Yafaev, D.R., Mathematical scattering theory, American Mathematical Society. 1992. 